Paper accepted for publication by Journal of Rail and Rapid Transit, 18 June 2017

\title{
Prediction of rail and bridge noise from concrete railway viaducts using a multi-layer rail fastener model and a wavenumber domain method
}

\author{
Qi $\mathrm{Li}^{1}$, David J Thompson ${ }^{2}$
}

\begin{abstract}
Concrete viaducts are an important part of the urban rail transit system but they produce considerable noise pollution in the communities nearby. The vibration generated at the wheel-rail interface is transmitted along the rail and also into the bridge so that noise is radiated from both the rail and the bridge. To facilitate noise predictions it is desirable to develop a model that takes into account the vibration generation and transmission in the traintrack-bridge system. The vibration and the associated noise of the track-bridge system are computed with a unified vibro-acoustic model using a wavenumber domain finite element and boundary element method. An important aspect is shown to be the frequency-dependent stiffness of a typical rail fastener utilized on bridges due to resonance of the baseplate between two rubber pads. In this study a multi-layer fastener model is proposed to allow for this effect. The proposed procedure is applied to a viaduct with a U-shaped section and compared with field measurements during train pass-bys. The elastic modulus and damping of the rubber pads and equivalent loss factor of the rail are chosen by fitting the calculated track decay rates to those estimated from measured rail accelerations under train passages. The wheel-rail combined roughness is also derived from the measured rail vibration. A comparison is then made between the simulated and measured bridge vibration to verify the proposed method as well as the parameters used in the track-bridge system. The predicted noise levels are also compared with the measured results. The effects of the fastener model, fastener stiffness, bridge damping and interference between multiple wheels are then discussed. It is found that the bridge noise has a non-negligible effect on the total A-weighted
\end{abstract}


noise levels in the region beneath the bridge and up to $30 \mathrm{~m}$ away from the track.

\section{Keywords}

Concrete viaducts; noise and vibration; rail fastener; wheel-rail interaction; wavenumber domain method

${ }^{1}$ Department of Bridge Engineering, Tongji University, 1239 Siping Road, Shanghai 200092, China.

${ }^{2}$ Institute of Sound and Vibration Research, University of Southampton, Southampton SO17 1BJ, UK.

\section{Introduction}

The noise from early elevated urban rail transit systems in Europe, Japan and the US led to concerns that it may affect the health and well-being of residents living or working in the vicinity of the rail systems. This attracted Stuber ${ }^{1}$, Kurzweil ${ }^{2}$, Remington and Wittig ${ }^{3}$, Ungar and Wittig ${ }^{4}$ to carry out initial investigations on the characteristics, mechanism and prediction of noise from elevated systems. More recently, understanding in this field has been developed by many researchers. A detailed review is given by Li et al. ${ }^{5}$. Unfortunately, many noise complaints still arise in the community near concrete elevated rail systems, even where some countermeasures have been applied for noise control. The vibration generated at the wheel-rail interface is transmitted along the rail and also to the bridge through the rail fasteners that connect the rail into the bridge. The noise radiation is caused not only by the vibration of the concrete bridge but also by that of the rail ${ }^{6}$. They are each dominant in a particular frequency range below $1000 \mathrm{~Hz}$ and in a particular spatial zone, with the rail radiation being more important above the bridge and less important beneath it. The wheel noise also plays a role at higher frequency. To assist the understanding and control of noise from elevated railway structures, a suitable noise prediction model of the coupled trackbridge system is required that accounts for the vibration transmission from the rail to the bridge as well as the sound radiation from these two components of the system. 
Existing models for the prediction of noise from railway bridges often divide the problems into several steps. Kurzweil ${ }^{2}$ briefly described a model for bridge vibration and noise prediction using statistical energy analysis (SEA). Remington and Wittig ${ }^{3}$ developed a similar model for noise prediction with three steps: the excitation of rail vibration through a mobility-based wheel-rail interaction technique; the transmission of vibration through an SEA-based rail-tie-girder interaction model; and the radiation of sound based on the radiation efficiency of each component of the wheel-track-bridge system. Janssens and Thompson ${ }^{6}$ also proposed an SEA-based bridge noise prediction model in which the power input to the bridge was calculated from the bridge mobility. This allowed a more detailed model of the rail fastening system to be considered using a multi-layer structure, as introduced by Bewes ${ }^{7}$ and Herron ${ }^{8}$. The SEA-based techniques were also utilized and improved by Hardy ${ }^{9}$, Harrison et al. ${ }^{10}$, Bewes et al. ${ }^{11}$, and recently by Li et al. ${ }^{12}$ and Zhang et al. ${ }^{13}$.

SEA-based methods are very attractive and effective for medium-to-high frequency vibration and noise prediction of large bridge structures consisting of many components. Nevertheless, numerical methods using finite element (FE) and boundary element (BE) techniques are more desirable to understand the detailed mechanisms of noise generation and propagation. The latter become more practical as computing power increases. Walker et al. ${ }^{14}$, and Crockett and Pyke ${ }^{15}$ used the FE method to calculate the vibration of bridges, but they just made a simple estimate of structure-borne noise based on the derived vibration of each bridge component. The direct use of the FE and BE methods in noise predictions was adopted by Zhang et al. ${ }^{16}$ and $\mathrm{Wu}$ and Liu ${ }^{17}$. Li et al. ${ }^{18}$ utilized the mode superposition method in a three-dimensional (3D) FE analysis to calculate the dynamic responses of the train-trackbridge system and used the modal acoustic vector in a 3D BE analysis for the prediction of low frequency bridge noise. The sound radiation part of this method has been improved significantly by $\mathrm{Li}$ et al. ${ }^{19}$ using the 2.5-dimensional (2.5D) BE technique that obtains 3D 
results from a wavenumber transform of the solutions of the two-dimensional (2D) $\mathrm{BE}$ model. This 2.5D technique allowed for the prediction of noise from several spans of a simply supported bridge ${ }^{20}$ and the consideration of both rail and bridge noise up to $1 \mathrm{kHz}{ }^{21}$. To increase the frequency range in the prediction of rail and bridge noise, $\mathrm{Li}$ et al. ${ }^{22}$ also succeeded to use a $2 \mathrm{D}$ vibro-acoustic model with the combination of the vibration power from a coupled wheel-track-bridge model.

The support structure for the steel rail is generally a multi-layer system, regardless of whether ballasted track or ballastless track is considered. The behaviour of the multi-layer structure is strongly frequency-dependent due to its internal mass between resilient elements (e.g. rubber pads) and to a lesser extent the frequency-dependence of the rubber pads themselves ${ }^{23,24}$. Directly fastened track is a common and economical type of ballastless track widely used on elevated urban rail transit systems. However, in the existing models for vibration and noise prediction the direct fastening system is usually simplified as a single resilient layer of constant stiffness. Janssens and Thompson ${ }^{6}$ applied a model of two infinite Euler-Bernoulli beams connected by a single resilient layer, developed by Pinnington ${ }^{25}$, to calculate the power transmitted to the bridge from the rail excited by a point force. For the prediction of low frequency noise from the bridge, Li et al. ${ }^{18,19}$, Zhang et al. ${ }^{13,16}$ and Song et al. ${ }^{20}$ utilized a discrete spring model for the direct fastening system between the rail and bridge to obtain the vibration of bridge induced by the moving trains. The stiffness of the fastening system was assumed to be constant for frequencies below $250 \mathrm{~Hz}$. A similar fastening model was also applied by Li et al. ${ }^{22}$ and Song et al. ${ }^{21}$ for the medium-frequency noise prediction up to $1000 \mathrm{~Hz}$. The use of a single layer fastening model of constant stiffness might be appropriate for low frequency vibration analysis but may be less appropriate for medium-to-high frequency vibration. Herron ${ }^{8}$ noted the limitation of the single layer model of the track on bridges and proposed a model including a rigid mass layer and two resilient 
layers for directly fastened track similar to the model for ballasted track.

The wheel-rail interaction model is another important aspect in the noise and vibration prediction. All the wheels of a train on the same rail are actually coupled to each other through their interaction with the rail. This effect is obviously included in the time domain computation of wheel-rail interaction such as in reference ${ }^{21}$. In the frequency domain analysis, the interference of the wheels is generally realized by considering one wheel as the active wheel and the others as passive wheels ${ }^{26}$. Repeat calculations are required to obtain the contributions from all the wheels by taking each wheel as the active wheel in turn. Wu and Thompson ${ }^{26}$ have showed that the rail vibration induced by multiple wheels can be treated as incoherent sources in frequency bands above $20 \mathrm{~Hz}$. Therefore, the total vibration of the rail can be calculated using the principle of energy superposition. Wu and Thompson ${ }^{27}$ also found that the interference effect of multiple wheels on ballast track is insignificant for noise prediction in one-third octave bands. This is true when the track decay rate (TDR) is high in the case of a ballast track. However, for directly fastened track on viaducts it is more important to include the effect of multiple wheels because the TDR in a certain frequency range can be very low.

The accuracy of noise predictions depends on the fidelity of the models that represent the coupled wheel-track-bridge system, and the parameters used in the models. The numerical or analytical methods used also determine the accuracy and efficiency of the noise predictions. The aim of this study is to improve the vibration and noise prediction for a concrete bridge by introducing three improvements. Firstly, the vibration and noise are predicted in a unified model including an FE model for the vibration of the track-bridge system in the wavenumber domain and a corresponding coupled BE model for the sound radiation. The 3D results in the spatial domain are obtained from a Fourier transform over wavenumber. The prediction method is introduced in the Section 'Vibration and noise 
prediction method'. Secondly the direct fastening system is represented by a multi-layer orthotropic structure representing the rail pad, the baseplate and the baseplate pad. This takes into account the internal resonance of the steel baseplate between the two rubber pads and thus improves the estimates of the vibration transmission to the bridge. This is also introduced in the Section 'Vibration and noise prediction method' and its effect is discussed in later sections. Thirdly, as described in the Section 'Estimation of track parameters and wheel-rail combined roughness', the rail accelerations measured during train passages are used to obtain the elastic modulus and damping of the rubber pad, the equivalent damping of the rail and the wheel-rail combined roughness. These features are illustrated in a case study described in the Section 'Case study'. The measured vibration and total noise are compared with those computed using three possible sets of track parameters to obtain the best estimate. Results are presented to illustrate the accuracy of vibration and noise predictions in various frequency ranges. Finally in the Section 'Discussion of modelling assumptions and parameter selection' the effects of a number of assumptions in the model are discussed. A comparison is made between the measured results and the vibration and noise predicted using the multilayer fastening model and a single layer model. Moreover, an investigation is conducted into the effect on the bridge vibration and noise of the bridge damping and the interference between multiple wheels. With the help of the prediction model, the rail and bridge noise are separated from each other and their contributions are compared at different field points around the bridge.

\section{Vibration and noise prediction method}

Assumptions for the vibration analysis

A continuously welded rail is a good example of a waveguide structure ${ }^{28}$ which can be regarded as infinite in length in the longitudinal direction and with a constant cross-section. According to the WFE method ${ }^{28}$ this kind of structure can be modelled by a $2 \mathrm{D}$ mesh 
representing the cross-section while allowing for wave propagation in the third direction.

The support structure of rails on elevated bridges adopting a direct fastening system usually comprises rail pads, baseplates, and baseplate pads attached to the bridge (see Fig. 1). Although the track is effectively infinite, these components are generally discontinuous along the track direction. The WFE method can only be applied to the support structure of the rails with the following simplifications and assumptions: (1) the bearings, piers and foundations of the bridge are omitted; (2) the rail pads, baseplates, and baseplate pads are modelled by continuous layers with parameters per unit length equivalent to those of the discrete fasteners; (3) a bridge structure that consists of multi-span simply-supported girders is also assumed to be a waveguide structure connected to the rail through the fastening system. The first assumption is generally acceptable; they only have a significant influence on the vibration of the system below about $32 \mathrm{~Hz}^{18}$. The second one applies in the frequency range below the pinned-pinned resonance frequency of the rail around $1000 \mathrm{~Hz}$; in any case for soft fasteners the pinned-pinned effect is not usually significant ${ }^{29}$. The last assumption is generally acceptable if the waves in the bridge are attenuated at a high rate, so the wave reflections from the ends of the span are less important. This is not always true for a girder bridge at low-to-medium frequencies; the validity of this assumption will be discussed later by comparing the predicted noise with measurements.

Fig. 2 shows an example of a WFE model of a track-bridge system considered later in the case study. As well as the rail and the bridge structure, the model comprises three further layers of waveguide structure representing the rail pad, baseplate and baseplate pad, as shown in Fig. 2(b). Since the rail fasteners are not continuous but discretely distributed along the rail, no propagating wave in the longitudinal direction can exist in the real fastening systems. To consider this effect, the fastener layers are assigned orthotropic properties with extremely small stiffness in the longitudinal direction to neglect wave propagation in these layers in this 
direction.

Since the train speeds are generally far smaller than the wave propagation speeds along the rail, the Doppler effect can be ignored in the calculation of the rail vibration. The moving roughness method ${ }^{26}$ in the frequency domain is applied to excite the wheel-track-bridge system. The vibration responses of the track-bridge system at a given longitudinal position are calculated for different train positions relative to this fixed position. For example, the train is set to move towards the given position from a position 40ce different wavelengths occur in the two waveguide structures. It would be possible to consider the com $\mathrm{m}$ away from it with a step of $0.2 \mathrm{~m}$ until the whole train has passed this position. The combined roughness corresponding to each wheel on the two rails is assumed to be incoherent, which is reasonable for roughness wavelengths less than about $4 \mathrm{~m}^{30}$. The vibration and noise induced by roughness excitations on the two rails are thus treated independently. Because the TDRs for the tracks on viaducts are usually quite low, it is important to take account of the interference among all the wheels through the interaction with the rail. This is explained further in the Section 'Wheel-rail interaction'. As the flexible modes of the wheel usually play an important role only for frequencies above $2 \mathrm{kHz}{ }^{29}$, a rigid wheel model based only on its mass is adopted as an approximation. Only vertical wheel-rail interaction is considered in the present model, although it can readily be extended to the lateral direction.

\section{Assumptions for the acoustic analysis}

Many field tests have shown that the dominant frequency of the A-weighted noise from elevated urban rail transit bridges is between about 600 and $800 \mathrm{~Hz}$. In this frequency range the rail is important as well as the bridge. This study therefore mainly investigates the noise from the rail and the bridge below $1000 \mathrm{~Hz}$. The wheel noise is not considered since it is generally significant in the rolling noise only above $2 \mathrm{kHz}{ }^{29}$. The noise radiated from the vehicles is also neglected in the simulation but the noise scattering effect of the car bodies is 
included in the model.

A wavenumber boundary element (WBE) method ${ }^{28}$ is coupled with the WFE method to obtain the exterior noise radiated from the waveguide structures subjected to given loadings. To form the coupled wavenumber finite element and boundary element (WFE-BE) model, both the WFE and WBE meshes (or sub-models) are established separately, and then the coupling condition between the solid and fluid elements is assigned to fulfil Dirichlet boundary conditions ${ }^{28}$. The calculations are carried out using the WANDS program ${ }^{31}$. This is a $2.5 \mathrm{D}$ method that is well-suited to the calculation of rail noise. The method is also wellsuited to predictions of the noise from long viaduct structures as the geometry is effectively constant over the length. In fact $\mathrm{Li}$ et al. ${ }^{22}$ has shown that a $2 \mathrm{D}$ acoustical model can give acceptable accuracy for the rail and bridge noise during the pass-by time in the mediumfrequency range $(200 \mathrm{~Hz}-1000 \mathrm{~Hz})$, as long as the spatially-averaged structural vibration is obtained accurately. The representation of the bridge noise by a $2.5 \mathrm{D}$ model additionally considers the noise variation along the longitudinal direction. The noise from rail pads, baseplates, baseplate pads and the clips is omitted because their vibration levels and radiation areas are generally less than that of the rail.

\section{Vibration and sound radiation under point harmonic force}

A WFE model is first developed (see Fig. 2) with 8-node quadratic solid elements to account for bending, shearing and cross-sectional deformation of the track-bridge system of infinite length. A point harmonic force of unit amplitude is applied on each of the rail heads to represent the wheel-rail contact force. The velocity response $\tilde{Y}_{m}(\kappa)$ of a given node $m$ can be computed in the WANDS program ${ }^{31}$ at each wavenumber $\kappa$ and the given circular frequency $\omega$. The velocity responses $Y_{m}(x)$ in the spatial domain as a function of distance from the force location can be then obtained through inverse Fourier transformation. 


$$
Y_{m}(x)=\frac{1}{2 \pi} \int_{-\infty}^{\infty} \tilde{Y}_{m}(\kappa) e^{-j \kappa x} d \kappa
$$

where $j=\sqrt{-1}$. Eq. (1) can be calculated from the discrete inverse Fourier transformation or a simple numerical integration method. However, these methods can result in unrealistic solutions near the force point due to the ideal point force and errors introduced by the truncation of the wavenumber range ${ }^{28}$. The residue method ${ }^{28}$ is therefore utilized in this study as an alternative way to calculate the spatial responses based on the eigenvalue analysis of the free wave equation for the WFE model.

To calculate the exterior noise field, a WBE mesh is then established including the profile of the rail, bridge and car body (see Fig. 3), using 3-node quadratic boundary elements. Two separate calculations are performed. In the first, the noise due to the vibration of the rail is calculated by coupling the WFE elements of the vibrating rail to the corresponding WBE elements and assigning all other surfaces rigid boundary conditions to allow for scattering of sound. In the second the same procedure is used to calculate the noise from the bridge. The sound pressure at a given field point can be obtained from the WFE-BE model at each calculated wavenumber and frequency. Here the residue method cannot be used so a simple numerical integration is used with a truncation of the wavenumber at a maximum value $\kappa_{\max }$. The sound pressure in the spatial domain for a certain frequency $\omega$ can be then obtained as

$$
P_{l}(x)=\frac{1}{2 \pi} \sum_{\kappa=-\kappa_{\max }}^{\kappa_{\max }} \tilde{P}_{l}(\kappa) e^{-j \kappa x} \Delta \kappa
$$

where $\Delta \kappa$ denotes the wavenumber step in the WFE-BE calculation; $\tilde{P}_{l}(\kappa)$ is the sound pressure at the given field point $l$ in the wavenumber domain, produced by unit harmonic force at frequency $\omega$, and $P_{l}(x)$ is the sound pressure of the field point in the space domain with a longitudinal distance of $x$ relative to the position of the force. 
In this study, all the WFE and WFE-BE calculations and the following wheel-rail interaction analysis as well as vibration and noise predictions are firstly carried out at oneninth octave frequency spacing (i.e. 30 points per decade), and the computed results are finally converted to one-third octave band resolution for presentation.

\section{Wheel-rail interaction}

To take account of the interaction between the multiple wheels of a whole train on the same rail, the roughness at each wheel-rail contact point is assumed to be incoherent with the others, which is valid above about $20 \mathrm{~Hz}$ in one-third octave bands ${ }^{26}$. The wheel-rail contact forces induced by the combined roughness can be obtained by considering one wheel as the active wheel with roughness excitation and treating the others as passive wheels coupled to the track without roughness excitation ${ }^{26}$. Treating the $i$ th wheel as the active one, an effective roughness of complex amplitude $R_{i}$, including the contact filter effect ${ }^{29,32}$, is introduced at this wheel-rail interface. The wheel-rail interaction is then determined through a matrix formulation of the compatibility of the wheel-rail displacement as

$$
\left[\mathbf{Y}_{\mathrm{rh}}+\mathbf{Y}_{\mathrm{c}}+\mathbf{Y}_{\mathrm{w}}\right] \mathbf{F}_{\mathrm{c}}^{i}=j \omega\left[\begin{array}{llllll}
0 & \cdots & 0 & R_{i} & \cdots & 0
\end{array}\right]^{\mathrm{T}}
$$

where $\mathbf{F}_{\mathrm{c}}^{i}$ represents the wheel-rail contact forces at all the wheels in the vertical direction induced by the combined roughness at the $i$ th wheel-rail interface; the element $Y_{\text {rh }}\left(x_{i k}\right)$ in $\mathbf{Y}_{\mathrm{rh}}$ stands for the transfer mobility of the rail head corresponding to the longitudinal distance $x_{i k}$ between the $k$ th and $i$ th wheels, which is computed from the WFE model; $\mathbf{Y}_{\mathrm{c}}$ is a diagonal matrix containing the mobility of the linearized Hertzian contact spring; $\mathbf{Y}_{\mathrm{w}}$ is a diagonal matrix of the wheel mobilities.

It is assumed that the roughness at each wheel $R_{i}$ is incoherent ${ }^{26}$ and has the same spectrum, thus the total amplitude of wheel-rail contact force $F_{\mathrm{c} n}$ at the $n$th wheel, caused by roughness at all the wheels, can be expressed as the energy summation of the incoherent 
excitations

$$
F_{\mathrm{c} n}=\sqrt{\sum_{i=1}^{N}\left|F_{\mathrm{c} n}^{i}\right|^{2}}
$$

where $N$ is the number of wheels of the train on the rail; and $F_{\mathrm{c} n}^{i}$ is an element in vector $\mathbf{F}_{\mathrm{c}}^{i}$ denoting the wheel-rail contact force at the $n$th wheel due to roughness excitation at the $i$ th wheel. The wheel-rail contact forces under each wheel in the given frequency band are generally different for each position of the wheels. Nevertheless, the wheel-rail contact forces do not vary with the motion of the train as the transfer mobilities of the rail are assumed not to vary.

Vibration and noise during a train pass-by

As the vibration produced by each wheel is assumed to be incoherent, the root mean square (rms) value of acceleration $a_{m}$ at a given position on the track-bridge system can be then expressed by

$$
a_{m}=\frac{\omega}{\sqrt{2}} \sqrt{\sum_{n=1}^{N} F_{\mathrm{c} n}^{2}\left|Y_{m}\left(x_{n}\right)\right|^{2}}
$$

where $x_{n}$ is the longitudinal distance between position of interest on the actual track-bridge system and the $n$th wheel; and $Y_{m}\left(x_{n}\right)$ is obtained from the WFE model with a unit force on the rail, as explained in Section 'Vibration and sound radiation under point harmonic force'. It should be noted that the transfer mobility $Y_{m}\left(x_{n}\right)$ at the fixed node $m$ corresponding to the moving force (wheel) positions can be obtained from the model with the driving force at a fixed position on the rail using the reciprocity principle. By changing the wheel positions $x_{n}$ relative to the given location, the instantaneous vibration of the track-bridge system can be obtained at every step representing the motion of the train.

With the calculated wheel-rail contact forces $F_{c n}$ and the sound radiation $P_{l}\left(x_{n}\right)$ of the 
track-bridge system obtained for a unit force, the rms value of sound pressure $p_{l}$ at a given field point caused by all the wheels on a rail can be similarly obtained as a function varying with the 'motion' of the train along the rail.

$$
p_{l}=\frac{1}{\sqrt{2}} \sqrt{\sum_{n=1}^{N} F_{\mathrm{c} n}^{2}\left|P_{l}\left(x_{n}\right)\right|^{2}}
$$

\section{Estimation of track parameters and wheel-rail combined roughness}

The critical parameters governing the vibration of wheel-track-bridge systems are the wheel-rail combined roughness ${ }^{29,33}$, the TDR ${ }^{29,34}$ and other dynamic parameters such as the stiffness and damping of the rail pads. These critical parameters are estimated from field tests. As a result, the influence of parameter uncertainties can be reduced in the noise prediction.

\section{Track decay rate}

The measurement of the TDR has been standardised in EN 15461: $2008^{35}$ using the hammer impact method. An alternative way to measure it is the train pass-by method developed by Janssens et al. ${ }^{33}$ and Dittrich et al. ${ }^{36}$. In the current work this is applied to the TDR on a bridge. According to this method, the TDR $D$ is given by

$$
D=8.686 \beta
$$

where the related parameter $\beta$ is given by ${ }^{36}$

$$
\beta=\frac{-\ln \left(1-\frac{E_{L_{1}} N}{E_{L_{2}} \psi}\right)}{L_{1}}
$$

where $E_{L_{1}}$ is the summation of the measured rail vibration 'energy' (integration of the square of the rail vibration) at the sensor position during the passage of each of the wheels of a train over a small distance $L_{1}$ (less than the minimum distance between two wheels) centred at the sensor position; $E_{L_{2}}$ is the corresponding energy determined over the total pass-by time of the 
train. $E_{L_{1}}$ and $E_{L_{2}}$ can be calculated in each frequency band from the measured rail vibration. The parameter $\psi$ denotes the ratio of the squared rail vibration produced by all the wheels on the rail to that caused by the single wheel passing over the sensor position. It is given by ${ }^{36}$

$$
\psi=\sum_{n=1}^{N} \sum_{i=1}^{N} e^{-2 \beta\left|x_{i n}\right|}
$$

where $x_{i n}$ is the distance between the $n$th and ith wheels. As $\psi$ is a function of $\beta$, Eq. (8) has to be solved iteratively. It is therefore called the 'energy iteration method' by Dittrich et al. ${ }^{36}$.

Eq. (8) allows the estimation of the TDR without a detailed knowledge of the track, the train or the roughness. However, two assumptions are made in this inverse method for the estimation of TDR. Firstly, the interference between wheels is neglected. Secondly, it is assumed that the dependence of the rail vibration induced by each wheel on the distance along the rail can be described by a decaying exponential function (Eq. (9)).

According to its definition another way to estimate the TDR is to obtain the slope of the rail vibration level curve over a certain distance $L_{3}$ before or after the passage of a wheel. To apply this method, the rail acceleration levels in each frequency band should be obtained at short time intervals corresponding to the motion of the train by steps of e.g. $0.1 \mathrm{~m}$. The time instant must be also detected when the relevant wheel is just passing over the sensor position on the rail. Then the linear fitting can be conducted on the time-history of the rail acceleration levels over the distance $L_{3}$ starting from the detected time instant. The TDR is simply the absolute value of the fitted slope. This method is referred to here as the 'slope fitting method'.

The energy iteration method and slope fitting method are both used in the Section 'Case study'.

\section{Pad stiffness and rail damping}

The values of the stiffness and damping parameters of the track support are required for 
use in the numerical models. The measured rail mobilities from the impact hammer measurements can be used to determine the rail fastener stiffness and damping loss factor by fitting the calculated mobilities to the measured results ${ }^{37}$. When the measured mobilities are not available, the estimated TDR can alternatively be used to derive these parameters with a similar curve fitting method.

At low frequency the TDR is generally high as the waves are blocked by the support stiffness ${ }^{29}$; they may also be affected by the stiffness of the bridge structure beneath the fasteners. In the medium-frequency range, the TDR drops suddenly at a frequency that depends on the fastener stiffness. Meanwhile, at higher frequencies the model requires a damping loss factor to be added to the rail to give good agreement with the measured values 29. According to this principle, the stiffness of the fastening system can be chosen to fit the measured TDR in the region where it drops, its loss factor can be chosen to match the extent of the drop and the damping loss factor of the rail can be selected to fit the TDR at high frequency. Finally, the TDR should be simulated using the chosen parameters and checked against the measured result over a wide frequency range to ensure a good agreement with the measured curves.

It may be noted that the TDRs estimated from the pass-by methods are obtained with the train loading. The track stiffness and damping derived from the TDRs of the loaded track are more suitable for the prediction of bridge vibration and noise than those obtained from the unloaded track because the force transmission to the bridge is governed by the fasteners near the wheels. However, the rail vibration is generally influenced by the fasteners both near and far from the wheels. The use of a load-dependent stiffness model ${ }^{27}$ of the fastener might be more suitable for the vibration simulation but it is not included in this study.

\section{Combined roughness}

From Eqs (3)-(5) it can be seen that the magnitudes of the wheel-rail contact force and of 
the rail vibration in the vertical direction are both linearly dependent on the magnitude of the wheel-rail combined roughness. This relationship enables the estimation of the roughness from measured rail vibration. If the track parameters are determined, or at least estimated with reasonable accuracy, the rms value of wheel-rail combined roughness $r$ can be estimated from

$$
20 \log _{10} \frac{r}{r_{0}}=20 \log _{10} \frac{a_{m}}{a_{0}}-20 \log _{10} \frac{a_{s}}{a_{0}}+20 \log _{10} \frac{r_{s}}{r_{0}}
$$

where $a_{m}$ is the measured rms acceleration of the rail in each one-third octave frequency band; $a_{s}$ is the simulated rms acceleration obtained using the assumed roughness $r_{s}$ and given track parameters; $r_{0}$ and $a_{0}$ are reference values for the calculation of roughness level and vibration level respectively. It is noted that $r$ and $r_{s}$ are both expressed here as functions of frequency but can be readily expressed in terms of wavelength for a given train speed.

\section{Case study}

\section{Introduction to the field test}

An elevated bridge for urban rail transit line 16 in Shanghai was chosen to demonstrate the accuracy of the proposed method for vibration and noise prediction through comparison with field measured results obtained during the trial operation of this line. The test section was selected on a straight track comprised of many spans of simply supported U-shaped girders (see Fig. 2). The single track girder has a standard span of $30 \mathrm{~m}$, a height of $1.8 \mathrm{~m}$ and a width of $5.54 \mathrm{~m}$. The thickness of the bottom slab and webs is $0.25 \mathrm{~m}$. The track structure on the bridge is a kind of ballastless track comprised of bi-block concrete bearing blocks, steel rails and WJ-2A fasteners connecting the rail and the rail bearing blocks. The rail bearing blocks are cast in situ to integrate with the prefabricated U-shaped girder. Tables 1 and 2 list the parameters for the rail, rail fastener and bridge used in this study. In September 2013, a test train with three cars was dispatched to cross the test section with different speeds 
from $40 \mathrm{~km} / \mathrm{h}$ to $120 \mathrm{~km} / \mathrm{h}$. Fig. 4 shows the layout of eight accelerometers used to measure the vibration of the rail in the lateral (A1) and vertical (A2) directions, the vertical vibration of the baseplate (A3), the vertical acceleration of the rail bearing block (A4) and the accelerations at several positions normal to the surface of the U-shaped girder (A5-A8). Eight microphones (see Fig. 5) were installed to measure the sound pressure at positions beneath the bridge ( $\mathrm{N} 1$ and $\mathrm{N} 2), 7.5 \mathrm{~m}$ from the track centre $(\mathrm{N} 3-\mathrm{N} 5)$ and $25 \mathrm{~m}$ from the track centre (N6-N8). The positions N4 and N7 were $1.5 \mathrm{~m}$ above the rail head. Microphones at positions N5 and N6 were out of order during the tests.

\section{Models and parameters for the vibration model}

Fig. 6(a) depicts the estimated TDRs for vertical vibration obtained from the energy iteration method and slope fitting method averaged over six train passages (two passages at each speed: 60,80 and $100 \mathrm{~km} / \mathrm{h}$ ). The TDRs obtained from the two methods match well with each other in the high frequency range but have some discrepancies in the region where the TDR drops at around $200 \mathrm{~Hz}$. For comparison, the WFE model shown in Fig. 2 is used to compute the TDR. Tables 1 lists three sets of parameters for rail pads and baseplate pads (referred to as A, B and C) that are each chosen to fit the estimated track decay rates. Table 1 also gives the equivalent static stiffness of the pads and fasteners per unit length which is calculated based on the 2D plane stress model of the fastener similar to the WFE mesh shown in Fig 2(b). As the stiffness of the individual pads is not known, the results from these three plausible parameter sets are compared with the measurements to find the most reasonable one. Table 2 gives other parameters of the track and bridge used in this study. The parameters of track set A were chosen to match the averaged TDRs from the two pass-by methods by assuming the same material properties for the rail pad and baseplate pad. The parameters of track set $\mathrm{B}$ and $\mathrm{C}$ were selected to match the TDRs from the two pass-by methods in each case with a stiffer rail pad and a softer baseplate pad. It is noted that the equivalent static 
stiffness of set $\mathrm{C}$ is the closest to the static stiffness of the fastener provided by the product supplier, which is about $40 \mathrm{MN} / \mathrm{m}$ per fastener.

Fig. 6(b) shows the estimated TDR curves for the lateral direction together with the computed ones obtained with the three sets of parameters. Although the lateral rail vibration is not used directly, the lateral TDR offers additional information to evaluate the performance of the different parameter sets. The computed TDRs from track sets B and C agree better with the ones estimated from the measurements than that from track set $\mathrm{A}$ in the region where the TDR drops. The computed result from set A corresponds to a much lower lateral stiffness of the rail fastening system than would be estimated from the TDR. As a result, the parameter sets $\mathrm{B}$ and $\mathrm{C}$ are more likely to match the real situation for the fasteners installed on the test bridge.

To show the effect of the two-stage resilient support in the rail fastener system, Fig. 7(a) gives the driving point stiffness at the top of the rail pad for the three sets of track parameters. Here the base of the fastener is fixed rigidly. Fig. 7(b) shows the corresponding transfer stiffness which is the blocked force transmitted to the rigid base through the baseplate pad for a unit displacement at the top of the rail pad. These results were calculated using a plane stress 2D finite element model. It can be observed from the low frequency asymptotes in Fig. 7 that the equivalent stiffness per unit length for the three sets of parameters is close to the corresponding equivalent static stiffness of the fasteners listed in Table 1. At higher frequencies, both the point and transfer stiffness of the fastener have significant frequency dependence due to the resonance of the baseplate mass between the two rubber pads. Therefore, it is important to take into account this effect for the prediction of medium-to-high frequency vibration of the rail and bridge where this direct fastening system is used.

Using these different parameter sets, the rail mobilities were calculated from the WFE model shown in Fig. 2. These were then used to determine the rail vibration excited by a unit 
roughness amplitude at each frequency using Eqs (3-5). The effective combined roughness was then estimated using Eq. (10) from the measured rail vertical vibration level averaged over two train passages with a speed of $80 \mathrm{~km} / \mathrm{h}$. Fig. 8 shows the effective combined roughness obtained using the three different track parameter sets. It is clear that the use of different parameters will lead to differences in the estimated roughness levels. To determine which set of track parameters gives the best representation of the actual track, the computed bridge vibration will be compared with the measured one in the Section 'Verification of predicted vibration'.

\section{Models and parameters for the acoustic model}

The WBE model of the train-track-bridge system shown in Fig. 3(a) was used to calculate the noise radiated by the bridge and the rail. The density and sound speed of the air were taken as $1.21 \mathrm{~kg} / \mathrm{m}^{3}$ and $343 \mathrm{~m} / \mathrm{s}$, respectively. The maximum element size in the WBE model was $134 \mathrm{~mm}$ (giving 2.5 quadratic elements per acoustic wavelength at $1000 \mathrm{~Hz}$ ). A total of 728 field points were selected on 20 concentric circles with radii from $3 \mathrm{~m}$ to $31.5 \mathrm{~m}$ with a step size of $1.5 \mathrm{~m}$, as shown in Fig. 3(b). Each circle was centred at the track centre and comprised 36 field points. The eight measurement positions were also added to the field points. Another 728 field points were used to account for the ground reflection by the image source method.

To separate the bridge noise from the rail noise, four WFE-BE models in the wavenumber domain were established by coupling the WBE model with different parts of the WFE model. The first model was the noise calculation model for the right rail: the WBE elements for the right rail were coupled to the WFE elements of the right rail which was subjected to a unit harmonic force; all other WBE elements were allocated a fixed boundary condition. The second model was for the noise from the left rail and was assembled in a corresponding way. The last two models were for the noise from the bridge with unit forces 
acting on the left or right rails respectively. Having obtained the radiated sound due to a unit force, these results were then scaled by the interaction force spectra of multiple wheels according to Eq. (6) to get the actual sound radiation during the train passage. The noise radiated from these four models is regarded as incoherent; it is assumed that the roughness excitation on the two rails is incoherent ${ }^{30}$ while the rail and bridge are considered as incoherent noise sources ${ }^{6,22}$ since different wavelengths occur in the two waveguide structures. It would be possible to consider the combined radiation in a single model but in the present analysis it is possible to study their separate contributions. It has been verified that these two approaches give the same result. Therefore, the energy superposition principle can be applied to calculate the total noise from the two rails and from the bridge due to the input from the two rails.

\section{Verification of predicted vibration}

Fig. 9 shows the comparison between the measured acceleration levels and simulated ones at the five sensor positions (A4-A8) on the bridge. Note that the computed vertical vibration of the rail (A2) has been adjusted to match exactly the measured one through the estimation of the effective roughness. It can be seen from Fig. 9 that the computed vibration levels obtained using the three sets of track parameters generally agree with the measured ones in terms of overall tendency. In the medium frequency range from $200 \mathrm{~Hz}$ to $1000 \mathrm{~Hz}$, track parameter set $\mathrm{C}$ gives better results for the vibration on the rail bearing block (A4) and bottom slab (A5) while track parameter set B provides better results for the vibration of the webs of the U-shaped girder (A6 and A7). Below $80 \mathrm{~Hz}$, the bridge vibration calculated from the three track parameter sets is almost identical due to the strong coupling between the rail and the bridge. It can be observed that, apart from position A6, the bridge vibration in this frequency range is overestimated by 10 to $15 \mathrm{~dB}$ compared with the measured one. The reason for this discrepancy is unknown. However, it is noted that similar discrepancies were 
found using a different modelling approach ${ }^{22}$ suggesting that the problem may lie in the measured rail vibration.

Fig. 10 presents a comparison of the instantaneous vibration levels between the measured and predicted results for the fixed measurement positions on the rail (A2) and the bridge (A5) at frequencies $80 \mathrm{~Hz}, 200 \mathrm{~Hz}$ and $1000 \mathrm{~Hz}$. They were calculated every $0.2 \mathrm{~m}$ with the motion of the train that ran from left to right in the figure. The location $x=0$ indicates the arrival of the first wheel of the train at the middle span (measurement location) of the test girder. It can be observed from Fig. 10(a-c) that fluctuations of the predicted rail vibration agree well with the measured curve at these three frequencies. This shows that the proposed method gives a good prediction in terms of the variation of rail vibration, although it should be remembered that the average vibration level has been adjusted to match the measured result by the choice of equivalent roughness. However, the discrepancies between prediction and measurement become much larger for the bridge vibration (see Fig. 10(d-f)). The rise and fall of the predicted vibration levels with the motion of the train are more gradual than those of the measured ones, even when the average predicted vibration levels agree with the measured ones at $200 \mathrm{~Hz}$ (Fig. 10(e)). In addition, the bridge vibration is overestimated by the WFE model when the train is not on the test span, as the model allows the vibration to be transmitted along the bridge from positions far away from the measurement position. This effect is very significant at low frequency when the wavelength of the bridge is large and there is little attenuation of the vibration (see Fig. 10(d)). Nevertheless, this is not a crucial flaw of the WFE model if the focus is on the average vibration levels of the bridge during the pass-by time of the train.

In conclusion, the presented method can give a good prediction of the instantaneous vibration of the rail under a moving train. It can be used to obtain averaged bridge vibration levels during the pass-by time at higher frequencies between $200 \mathrm{~Hz}$ and $1000 \mathrm{~Hz}$, if the 
combined roughness is quantified from measured rail vibration but the bridge vibration is overestimated at low frequency.

Verification of predicted noise

Fig. 11 compares the A-weighted noise spectra between measured results at the six microphone positions (N1-N4, N7 and N8) and the predicted total noise levels obtained from the three sets of track parameters. It can be seen that the predicted noise levels at all the positions are about $15 \mathrm{~dB}$ larger than the measured ones below $80 \mathrm{~Hz}$. This is consistent with the fact that the vibration of the bridge is overestimated in this frequency range. The fastener parameters have little influence on the predicted noise below $80 \mathrm{~Hz}$ because the bridge vibration is hardly affected by the fastener parameters in this region, as mentioned in the Section 'Verification of predicted vibration'.

In the frequency range from 400 to $1000 \mathrm{~Hz}$ and for the field points above the bridge (N4 , N7 and N8), the predicted noise levels from the three sets of track parameters also show small differences $(0.4 \mathrm{~dB}-3.4 \mathrm{~dB})$ because the rail noise dominates the total noise and the rail vibration has been adjusted to the measured one through the estimated roughness. The predicted noise levels agree better with the measured ones in this frequency range compared with the results at the lower frequencies. This shows the acoustical model for rail noise prediction is of reasonable accuracy (better than $4 \mathrm{~dB}$ ) as long as the vibration is obtained accurately.

In the frequency range from 100 to $315 \mathrm{~Hz}$, it can be seen from Fig. 11 that the track parameter set $\mathrm{B}$ gives good noise prediction at positions $\mathrm{N} 1, \mathrm{~N} 2$ and $\mathrm{N} 3$ beneath the bridge but overestimates the noise for other measured positions above the bridge. For all six field points the track parameter set B gives up to $6 \mathrm{~dB}$ higher noise levels than those of parameter sets $\mathrm{A}$ and $\mathrm{C}$. This corresponds to the higher bridge vibration levels computed from set $\mathrm{B}$ (see Fig. 9) and shows that the bridge noise dominates the total noise in this frequency range for 
field points both beneath and above the bridge.

From the comparisons between the measured and predicted results in terms of the TDR, bridge vibration and total noise, it can be concluded that the track parameter set $\mathrm{C}$ gives the best agreement with the measured behaviour. However, the parameters of track set $\mathrm{C}$, and other parameters for the bridge, do not perfectly match the actual ones, and accordingly the discrepancies between the predicted and measured results cannot be reduced to a satisfactory degree. The influence of these parameters on the predictions of vibration and noise will be investigated in the following section. Moreover, the frequency region and spatial region in which the bridge noise and rail noise dominate will be further discussed.

\section{Discussion of modelling assumptions and parameter selection}

\section{Effect of fastening model and parameter}

In the WFE model introduced in the Section 'Vibration and noise prediction method', the rail fastener has been represented with two resilient layers and an intermediate baseplate. In this section the effect of introducing this model and the choice of stiffness values are investigated by comparing the results with those for a single resilient layer.

Fig. 12 shows a simple fastening model of constant stiffness used for the comparisons. Two values of the vertical stiffness of the fastening were chosen, set to $132 \mathrm{MN} / \mathrm{m}^{2}$ and 66 $\mathrm{MN} / \mathrm{m}^{2}$ by adjusting the elastic modulus of the rubber layer. These values were initially chosen to match the TDRs computed from the simple models around the frequencies where the TDR drops suddenly with those obtained from the multi-layer fastener models B and C. It has been seen from Fig. 7 and Table 3 that the multi-layer fastener models B and C have slightly smaller values of equivalent stiffness at low frequencies compared with the two corresponding simple fastener models.

Fig. 13 presents the differences in bridge vibration levels obtained at five measured positions (A4-A8) by using the multi-layer fastener model with parameter sets B and C, and 
the simple fastener model. The same effective roughness is assumed for all the models. Fig. 13(a) and Fig. 13(b) show the differences between the simple fastening model and the multilayer fastener model of the same low frequency stiffness. From these figures it can be observed that the simple pad model gives similar vibration predictions to the multi-layer fastener model below $315 \mathrm{~Hz}$ but gives lower bridge vibration (by around $8 \mathrm{~dB}$ ) at higher frequencies due to neglecting the resonance of the baseplate. Below $1 \mathrm{kHz}$ the differences are greater for the softer fastener (set C), especially around $500 \mathrm{~Hz}$, as shown in Fig. 13(b). At $3.15 \mathrm{kHz}$, the bridge vibration can be up to $10 \mathrm{~dB}$ larger when using the simple model compared with the multi-layer fastener model, due to the vibration isolation effect above the resonance frequency of the baseplate (see Fig. 7(b)). Fig. 13(c) and Fig. 13(d) show the differences in bridge vibration between parameter set $\mathrm{B}$ and $\mathrm{C}$ and between the corresponding simple fastening models. This shows that in both cases the vibration can be generally decreased by around $6 \mathrm{~dB}$ above $63 \mathrm{~Hz}$ by halving the fastener stiffness. However, the effect is less clear for the multi-layer fastener than for the simple fastening model.

Fig. 14 shows the difference in terms of bridge and rail noise levels at five measurement positions between the multi-layer fastener model with parameter set $\mathrm{C}$ and the corresponding simple fastener model with stiffness $66 \mathrm{MN} / \mathrm{m}^{2}$. The results of Fig. 14(a) are similar to the corresponding ones from the bridge vibration in Fig. 13(b), again showing large differences around $500 \mathrm{~Hz}$. Fig. 14(b) shows that the differences in rail noise levels have a more complex frequency dependence with differences of more than $+/-10 \mathrm{~dB}$.

From the above discussion, it can be concluded that the multi-layer fastener model used in this study gives similar vibration and noise predictions below $315 \mathrm{~Hz}$ to those obtained from the simple spring model of the fastener, but it allows for important frequency-dependent properties above $315 \mathrm{~Hz}$. Therefore it provides a better way to model the fastener than the simple model of constant stiffness. However, it still has some limitations for the vibration and 
noise prediction. It is well known that the fastener stiffness is generally dependent on the wheel loading on it. Although the equivalent fastener stiffness has been roughly estimated in this study based on the indirectly measured TDRs, it is difficult to quantify the exact stiffness of the fasteners with and without the wheel loading. Moreover, the clips and fastening bolts of the fastening system were not modelled. They can produce additional damping and stiffness in the fastener which can influence the vibration and noise from the track-bridge system. The measurement of fasteners installed in a test rig in the laboratory (see e.g. ${ }^{37}$ ) is required to obtain its driving point stiffness and transfer stiffness. The measurement of the separate properties of the rail pad and baseplate pad are also needed.

\section{Effect of interference of multiple wheels}

The effect of interference between multiple wheels has been be considered by Eq. (3) through the transfer mobilities of the rail. In order to show the influence of this aspect of the model, Fig. 15 shows the difference between the bridge vibration levels obtained from the model neglecting the coupling effect between the wheels and from that in which it is included. The track parameter set $\mathrm{C}$ was used in these calculations. It can be observed from Fig. 15 that there is very little effect below $200 \mathrm{~Hz}$, which is because the TDR is too high to allow for significant wheel interference (see Fig 6(a)). However, larger effects on both the track and bridge vibration can be seen, especially at $400 \mathrm{~Hz}$ and $630 \mathrm{~Hz}$, if the interference between wheels is ignored. The results for the rail and the bridge are very similar. Slightly smaller differences are found in the corresponding results for the stiffer parameter set B (not shown).

Fig. 16 presents the effect of neglecting the multiple wheel interaction on the noise from the rail and bridge. Similar changes of noise level can be observed to the differences in vibration level shown in Fig. 15. Since direct fastening systems on bridges often have low fastener stiffness, the interference effect becomes significant in the medium frequency range and thus should be considered in the predictions of vibration and noise from trains on bridges. 
Nevertheless, the interference effect depends strongly on the fastener stiffness, which is also load-dependent.

\section{Effect of bridge damping}

The damping of bridges over a large range of frequency is very difficult to obtain for real concrete girders because the damping is influenced by the concrete material itself, microcracks on the girder, bearings supporting the girder and items such as communication and power supply cables installed on the bridge. Damping can also be frequency-dependent. The use of inaccurate loss factors in the prediction can lead to disagreement with the measured results and may partly explain the differences observed earlier. Note that the mass and stiffness distributions of the measured bridge may not be homogeneous and orthotropic as assumed, but these are not discussed further. By way of illustration of the influence of damping, Fig. 17 shows the changes in bridge vibration and bridge noise levels obtained by increasing the loss factor of the bridge from 0.01 to 0.1 . It can be observed that a reduction of around $17 \mathrm{~dB}$ is found at around $1 \mathrm{kHz}$ for both bridge vibration and noise levels. The bridge damping has a more obvious effect on the vibration and noise at high frequencies than at low frequencies because vibration waves at low frequencies have longer wavelengths. It can be observed from Fig. 17(a) that the positions far from the rails (A6-A8) are more sensitive to the bridge damping than those near the rail (A4) because the former positions allow for more energy dissipation through a longer propagation path. If a smaller loss factor is assigned to the bridge in the frequency range from $200 \mathrm{~Hz}$ to $1000 \mathrm{~Hz}$, the predicted bridge vibration at positions A6, A7 and A8 could be made to match the measured one more closely whereas those at positions A4 and A5 would not be obviously changed.

\section{Comparison between rail and bridge noise}

Even though the track parameter set $\mathrm{C}$ listed in Table 1 has been carefully selected to give good agreement with the measurements, and the estimated roughness obtained from 
measured rail vibration has been adopted in the noise prediction, a number of factors remain that may lead to the discrepancies between predicted and measured noise: (1) inaccurate fastener parameters and bridge parameters will cause prediction errors in the bridge noise in the medium frequency range; (2) the estimated roughness represents the rail roughness near the test section but the radiated noise is determined by the rail roughness over a much longer distance, which may vary; (3) inaccurate modelling of the acoustical and vibrational boundary conditions also plays a role. Since so many factors are contributing to the noise prediction errors, it is difficult to adjust the models and parameters to give a good agreement. Fortunately, the discrepancies between the predicted and measured noise could be regarded as acceptable in the frequency range between $200 \mathrm{~Hz}$ and $1000 \mathrm{~Hz}$, particularly near the peak frequency $630 \mathrm{~Hz}$ (see Fig. 11). Therefore, the predicted results can be used to illustrate the spatial distributions of noise levels from the rail and bridge.

Fig. 18(a-c) presents contour maps of the rail, bridge and total noise field. Fig. 18(d) gives the contour map of total noise minus rail noise. It can be seen from Fig. 18(a) that the rail noise has more influence on the region above the bridge than beneath the bridge due to shielding by the parapet of the U-shaped girder. Fig. 18(b) indicates that the bridge noise has stronger noise radiation in the vertical direction than the lateral direction; although the vertical wheel-rail excitation dominates, both vertical vibration of the bridge deck and lateral vibration of the webs of the U-shaped girder are excited. Fig. 18(c) shows that the total noise above the bridge is similar to the rail noise shown in Fig. 18(a). Nevertheless, the total noise beneath the bridge is controlled by the combination of rail noise and bridge noise, as indicated by Fig. 18(d). This is because the rail noise reaching the region beneath the bridge by diffraction and scattering is comparable to that of the directly radiated bridge noise.

\section{Conclusions}

To improve the predictions of rail and bridge noise from elevated railways, a 
wavenumber finite element was utilized to model the rails, the fastening system and the multi-span bridge with waveguide structures of infinite length. A multi-layer fastening model has been proposed to allow for frequency-dependent point and transfer stiffness of the fastening system in the medium-to-high frequency range. The multiple wheels of a train on the rails were coupled together through the transfer mobilities of the rails and the compatibility condition of the wheel-rail relative displacement. The moving roughness model and the concept of active and passive wheels were applied to calculate the vibration of the wheel-track-bridge system in each frequency band with the motion of the train along the rail. The noise radiated from the rail and bridge was obtained from a wavenumber boundary element model that is coupled to the wavenumber finite element model. A U-shaped concrete bridge for which vibration and noise tests were conducted in the field was used as a case study to demonstrate the accuracy of the present prediction method. The parameters of the track were chosen to match the estimated track decay rates obtained from measured rail acceleration; the effective wheel-rail combined roughness was also obtained through the measured rail acceleration. The predicted vibration and noise were compared with the measured results. Further investigations were made on the influence of fastener model, track parameters, bridge damping and wheel interference effects. The dominant frequency range and spatial region of the bridge noise were finally obtained from the predicted noise field. The major conclusions of this study can be drawn as follows.

(1) The proposed wavenumber finite model of the track-bridge system with the multilayer fastener model can give a good prediction for the instantaneous fluctuation of the rail vibration. However, for the bridge vibration it is only possible to obtain correctly the averaged levels during pass-by time of the train, not the detailed evolution with time.

(2) The rail noise levels during the pass-by time can be well predicted when the track parameters and wheel-rail combined roughness are estimated from the measured rail 
vibration. However, the bridge noise below $80 \mathrm{~Hz}$ is overestimated due to an overestimation of the bridge vibration by the WFE model. The reasons for this are not clear and require further investigation. It is recommended to compare the directly measured wheel-rail combined roughness with the one estimated from the measured rail vibration, and to validate the fastener parameters through laboratory tests. Nevertheless, the proposed method can be applied to noise prediction between $200 \mathrm{~Hz}$ and $1000 \mathrm{~Hz}$ with satisfactory accuracy.

(3) The vibration and noise predictions of the track-bridge system are significantly affected by the fastener model and fastener parameters. The multi-layer fastener model provides a better way to model the fastening system than the simple model of constant stiffness. Nevertheless, the detailed properties of the multi-layer fastener should be investigated thoroughly, including the effects of loading.

(4) The interference effect of multiple wheels is significant in the medium frequency region since the direct fastening system on a bridge often has low values of fastener stiffness.

(5) The total A-weighted noise levels above the bridge are dominated by the rail noise, and those beneath the bridge are controlled by the combined effect of rail noise and bridge noise.

The frequency domain method used in this study provides an effective way to obtain rail and bridge noise below $1000 \mathrm{~Hz}$ during the pass-by time of the trains. Frequency-dependent stiffness and damping properties of rubber pads can be used in the rail fastener model if laboratory tests results are available in the future.

All data published in this paper are openly available from the University of Southampton repository at http://doi.org/10.5258/SOTON/D0135.

\section{Funding}

The study was supported by the China Scholarship Council, the Natural Science Foundation of Shanghai (No.15ZR1442800). 


\section{References}

1. Stuber C. Air-borne and structure-borne noise of railways. Journal of Sound and vibration. 1975; 43: 281-9.

2. Kurzweil L. Prediction and control of noise from railway bridges and tracked transit elevated structures. Journal of Sound and Vibration. 1977; 51: 419-39.

3. Remington PJ, Wittig LE. Measurement and analysis of the noise from and vibration in rapid transit elevated structures. The Journal of the Acoustical Society of America. 1980; 67: S62-S.

4. Ungar EE, Wittig LE. Wayside noise of elevated rail transit structures: analysis of published data and supplementary measurements. US Department of Transportation, Report No UMTA-MA-06-0099-80-6 (December 1980). 1980.

5. Li X, Yang D, Chen G, Li Y, Zhang X. Review of recent progress in studies on noise emanating from rail transit bridges. Journal of Modern Transportation. 2016: 1-14.

6. Janssens MHA, Thompson DJ. A calculation model for the noise from steel railway bridges. Journal of Sound and Vibration. 1996; 193: 295-305.

7. Bewes OG. The calculation of noise from railway bridges and viaducts. University of Southampton, 2005.

8. Herron D. Vibration of railway bridges in the audible frequency range. University of Southampton, 2009.

9. Hardy A. Noise from railway bridges. Proceedings of the Institution of Mechanical Engineers, Part F: Journal of Rail and Rapid Transit. 1999; 213: 161-72.

10. Harrison MF, Thompson DJ, Jones CJC. The calculation of noise from railway viaducts and bridges. Proceedings of the Institution of Mechanical Engineers, Part F: Journal of Rail and Rapid Transit. 2000; 214: 125-34.

11. Bewes OG, Thompson DJ, Jones CJC, Wang A. Calculation of noise from railway bridges and viaducts: experimental validation of a rapid calculation model. Journal of Sound and Vibration. 2006; 293: 933-43.

12. Li XZ, Liu QM, Pei SL, Song LZ, Zhang X. Structure-borne noise of railway composite bridge: Numerical simulation and experimental validation. Journal of Sound and Vibration. 2015; 353: 378-94.

13. Zhang X, Li XZ, Zhang JQ, Song LZ, Li YD. A hybrid model for the prediction of low-frequency noise emanating from a concrete box-girder railway bridge. Proceedings of the Institution of Mechanical Engineers, Part F: Journal of Rail and Rapid Transit. 2015: 0954409715605127.

14. Walker JG, Ferguson NS, Smith MG. An investigation of noise from trains on bridges. Journal of Sound and Vibration. 1996; 193: 307-14.

15. Crockett AR, Pyke J. Viaduct design for minimization of direct and structure-radiated train noise. Journal of Sound and Vibration. 2000; 231: 883-97.

16. Zhang X, Li X, Li X, Liu Q, Zhang Z. Train-induced vibration and noise radiation of a prestressed concrete box-girder. Noise Control Engineering Journal. 2013; 61: 425-35.

17. Wu T, Liu J. Sound emission comparisons between the box-section and U-section concrete viaducts for elevated railway. Noise Control Engineering Journal. 2012; 60: 450-7.

18. Li Q, Xu YL, Wu DJ. Concrete bridge-borne low-frequency noise simulation based on train-track-bridge dynamic interaction. Journal of Sound and Vibration. 2012; 331: 2457-70.

19. Li Q, Song XD, Wu DJ. A 2.5-dimensional method for the prediction of structure-borne low-frequency noise from concrete rail transit bridges. The Journal of the Acoustical Society of America. 2014; 135: 2718-26.

20. Song XD, Wu DJ, Li Q, Botteldooren D. Structure-borne low-frequency noise from multi-span bridges: A prediction method and spatial distribution. Journal of Sound and Vibration. 2016; 367: 114-28.

21. Song XD, Li Q, Wu DJ. Investigation of rail noise and bridge noise using a combined 3D dynamic model and 2.5 D acoustic model. Applied Acoustics. 2016; 109: 5-17.

22. Li Q, Li WQ, Wu DJ, Song XD. A combined power flow and infinite element approach to the simulation of medium-frequency noise radiated from bridges and rails. Journal of Sound and Vibration. 2016; 365: 134-56.

23. Fenander $\AA$. Frequency dependent stiffness and damping of railpads. Proceedings of the Institution of Mechanical Engineers, Part F: Journal of rail and rapid transit. 1997; 211: 51-62.

24. Wei K, Wang P, Yang F, Xiao JH. The effect of the frequency-dependent stiffness of rail pad on the environment vibrations induced by subway train running in tunnel. Proceedings of the Institution of Mechanical Engineers, Part F: Journal of Rail and Rapid Transit. 2014: 0954409714558438.

25. Pinnington R. Vibrational power transmission from a finite source beam to an infinite receiver beam via a continuous complaint mount. Journal of Sound and Vibration. 1990; 137: 117-29.

26. Wu TX, Thompson DJ. Vibration analysis of railway track with multiple wheels on the rail. Journal of Sound and Vibration. 2001; 239: 69-97.

27. Wu T, Thompson D. The effects on railway rolling noise of wave reflections in the rail and support 
stiffening due to the presence of multiple wheels. Applied Acoustics. 2001; 62: 1249-66.

28. Nilsson CM, Jones CJC, Thompson DJ, Ryue J. A waveguide finite element and boundary element approach to calculating the sound radiated by railway and tram rails. Journal of Sound and Vibration. 2009; 321: 813-36.

29. Thompson D. Railway noise and vibration: mechanisms, modelling and means of control: Elsevier, 2009.

30. Ntotsios E, Thompson DJ, Hussein M. Effect of rail unevenness correlation on the prediction of groundborne vibration from railways. Euronoise 2015, the 10th European Congress and Exposition on Noise Control Engineering. Maastricht, Netherlands, 2015.

31. Nilsson CM, Jones CJC. Theory manual for WANDS 2.1 wave number domain FE-BE software for structures and fluids. 2007.

32. Remington PJ. Wheel/rail noise-Part IV: Rolling noise. Journal of Sound and Vibration. 1976; 46: 41936.

33. Janssens MHA, Dittrich MG, De Beer FG, Jones CJC. Railway noise measurement method for pass-by noise, total effective roughness, transfer functions and track spatial decay. Journal of Sound and Vibration. 2006; 293: 1007-28.

34. Jones CJC, Thompson DJ, Diehl RJ. The use of decay rates to analyse the performance of railway track in rolling noise generation. Journal of Sound and Vibration. 2006; 293: 485-95.

35. CEN. EN 15461: 2008. Railway applications - noise emissions, characterization of the dynamic properties of track sections for pass by noise measurements. 2008.

36. Dittrich MG, Létourneaux F, Dupuis H. Background for a new standard on pass-by measurement of combined roughness, track decay rate and vibroacoustic transfer functions. Noise and Vibration Mitigation for Rail Transportation Systems: Springer, 2015, p. 197-204.

37. Thompson DJ, Verheij JW. The dynamic behaviour of rail fasteners at high frequencies. Applied Acoustics. 1997; 52: 1-17. 
Table 1 Parameters of the pads for various track sets

\begin{tabular}{|c|c|c|c|c|c|}
\hline Parameter set & $\begin{array}{l}\text { Rail pad elastic } \\
\text { modulus }\left(\mathrm{MN} / \mathrm{m}^{2}\right)\end{array}$ & $\begin{array}{l}\text { Baseplate pad } \\
\text { elastic modulus } \\
\qquad\left(\mathrm{MN} / \mathrm{m}^{2}\right)\end{array}$ & $\begin{array}{l}\text { Equivalent static } \\
\text { stiffness of rail pad } \\
\left(\mathrm{MN} / \mathrm{m}^{2}\right)\end{array}$ & $\begin{array}{c}\text { Equivalent static } \\
\text { stiffness of baseplate } \\
\operatorname{pad}\left(\mathrm{MN} / \mathrm{m}^{2}\right)\end{array}$ & $\begin{array}{l}\text { Equivalent static } \\
\text { stiffness of overall } \\
\text { fastener }\left(\mathrm{MN} / \mathrm{m}^{2}\right)\end{array}$ \\
\hline A & 15 & 15 & 92 & 388 & 70 \\
\hline B & 90 & 6.38 & 538 & 183 & 114 \\
\hline C & 60 & 2.92 & 362 & 88 & 62 \\
\hline \multicolumn{6}{|c|}{$\begin{array}{l}\text { Notes: } \\
\text { (a) the width, length and thickness of the rail pad are respectively } 151.5 \mathrm{~mm}, 190 \mathrm{~mm} \text { and } 10 \mathrm{~mm} \text {; the width, } \\
\text { length and thickness of the baseplate pad are respectively } 380 \mathrm{~mm}, 190 \mathrm{~mm} \text { and } 5 \mathrm{~mm} \text {; the mass of the pads is } \\
\text { omitted; } \\
\text { (b) the Poisson's ratio of the pads is set to be } 0.47 \text {, and the loss factor of the pads is set to be } 0.15 \text {; } \\
\text { (c) in the WFE model, the elastic modulus of the pads in the vertical and lateral directions is divided by a factor } \\
\text { of } 3.16 \text { to consider its length of } 0.19 \mathrm{~m} \text { within a fastener span of } 0.6 \mathrm{~m} \text {; } \\
\text { (d) the elastic modulus of pads in the longitudinal directions is divided by a factor of } 10 \text { to avoid } \\
\text { longitudinal wave propagation in the fastener layer. } \\
\text { (e) the equivalent mass of the baseplate per unit length is } 16.9 \mathrm{~kg} / \mathrm{m} \text {. } \\
\text { (f) the equivalent stiffness of the pads and fasteners is calculated based on the deformation of the pads } \\
\text { underneath the rail foot, considering the deformation of the baseplate. }\end{array}$} \\
\hline
\end{tabular}

Table 2 Parameters for other components of the track-bridge system

\begin{tabular}{ccccc}
\hline Parameter & Density $\left(\mathrm{kg} / \mathrm{m}^{3}\right)$ & Elastic modulus $\left(\mathrm{GN} / \mathrm{m}^{2}\right)$ & Poisson's ratio & Loss factor \\
\hline Rail & 7800 & 210 & 0.3 & $0.04(0.02)$ \\
Baseplate & 7800 & 210 & 0.3 & 0.15 \\
Rail bearing block & 2600 & 35 & 0.16 & 0.01 \\
Bridge deck & 3120 & 35 & 0.16 & 0.01 \\
Other part of bridge & 2860 & 35 & 0.16 & 0.01 \\
\hline
\end{tabular}

Notes:

(a) equivalent loss factor of the rail is 0.04 for track set $\mathrm{A}$ and 0.02 for sets $\mathrm{B}$ and $\mathrm{C}$.

(b) the density of the bridge takes account into the dead load of pavements and other facilities on the bridge;

(c) the width, length and thickness of the rail pad are respectively $380 \mathrm{~mm}, 190 \mathrm{~mm}$ and $18 \mathrm{~mm}$; in the WFE model, the density and elastic modulus of the baseplate in the vertical and lateral directions are divided by a factor of 3.16 to consider its length of $0.19 \mathrm{~m}$ within a fastener span of $0.6 \mathrm{~m}$;

(d) the elastic modulus of the baseplate in the longitudinal directions is divided by a factor of 10 to avoid longitudinal wave propagation in the fastener layer.

Figure 1. Typical structure of a rail fastener (WJ-2A) on urban rail transit bridges 


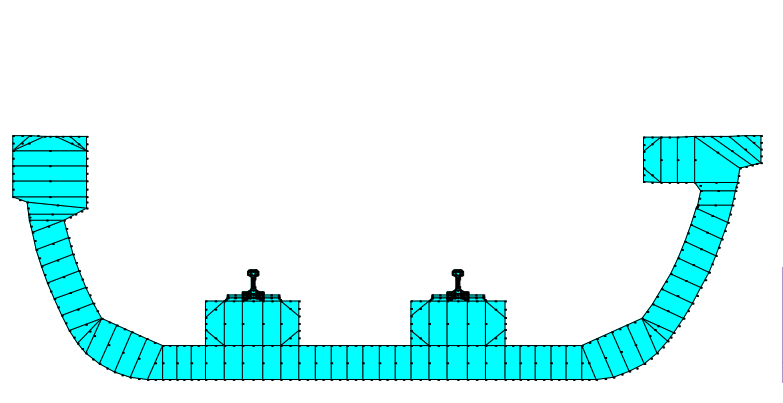

(a)

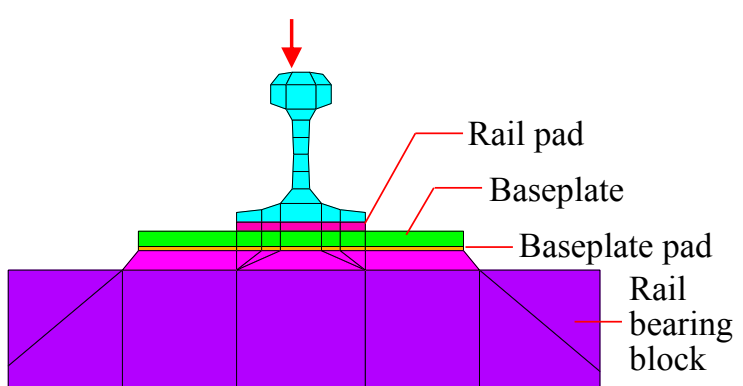

(b)

Figure 2. Model of track-bridge system for vibration analysis: (a) WFE mesh with quadratic elements; (b) zoom of (a), detail of multi-layer fastener model, middle nodes not shown

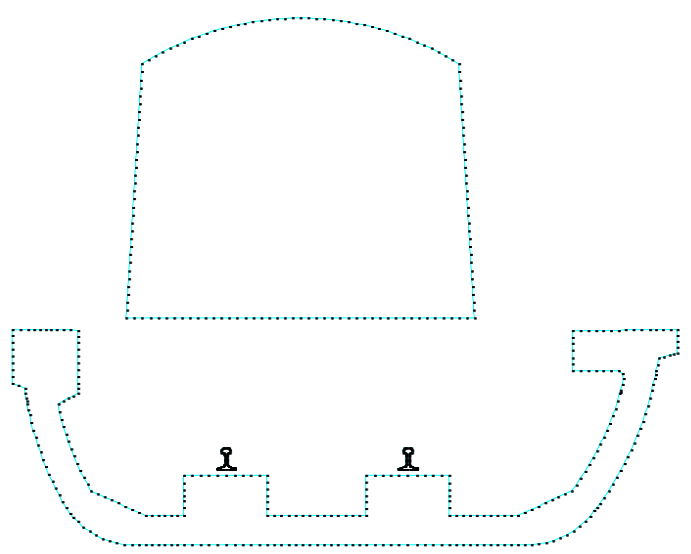

(a)

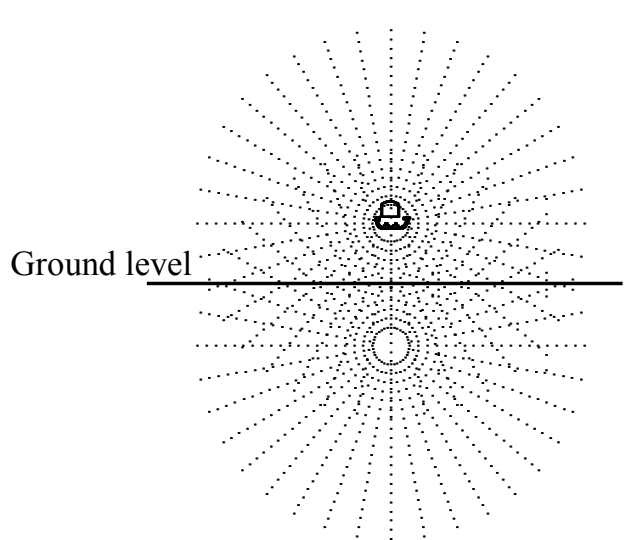

(b)

Figure 3. Model of train-track-bridge system for acoustic analysis: (a) WBE mesh; (b) field points

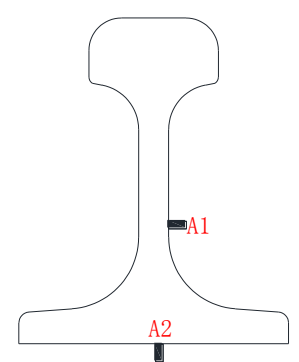

(a)

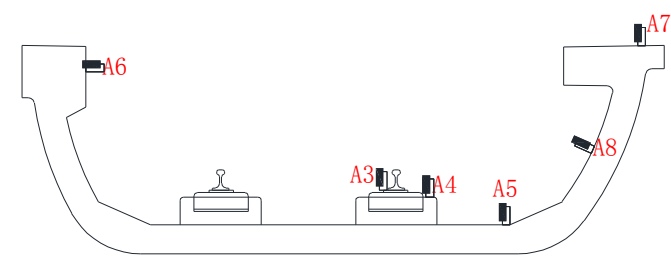

(b)

Figure 4. Layout of the accelerometers on the track-bridge system: (a) the rail; (b) baseplate and bridge 


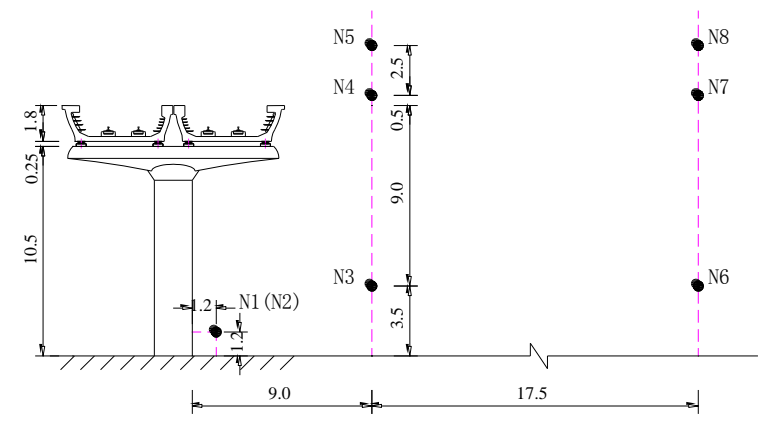

(a)

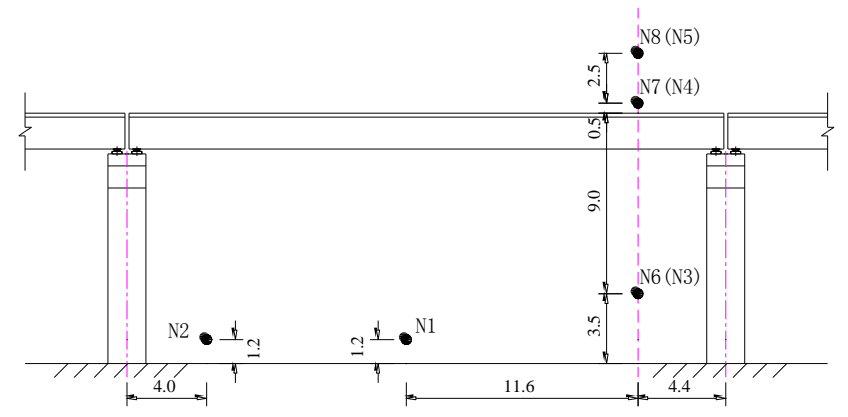

Figure 5. Layout of microphones (units: m): (a) section; (b) elevation view

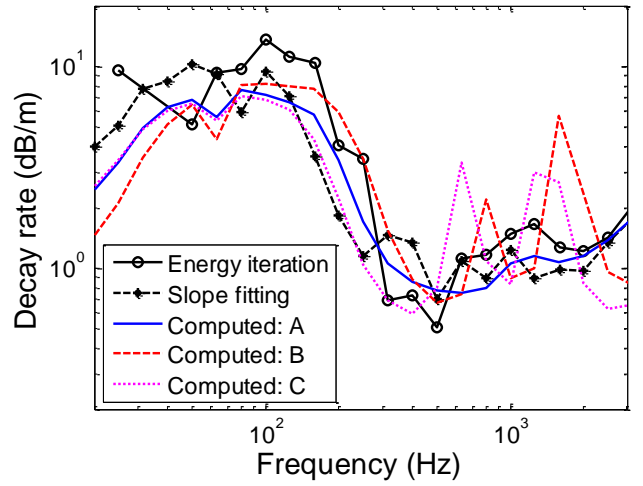

(a)

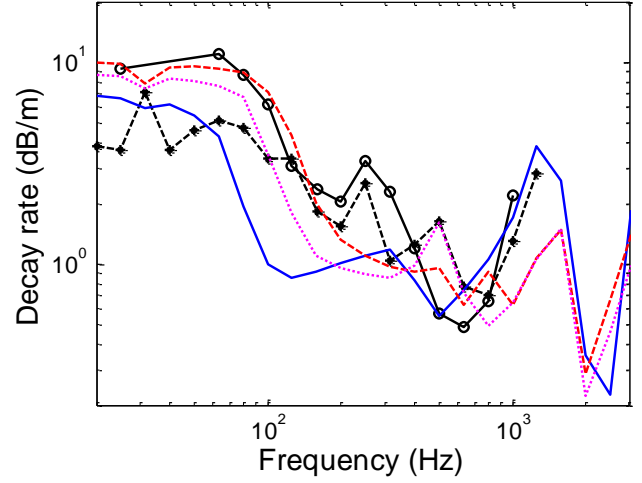

(b)

Figure 6. Estimated track decay rates from train pass-by methods and simulated ones using different track models: (a) vertical; (b) lateral 


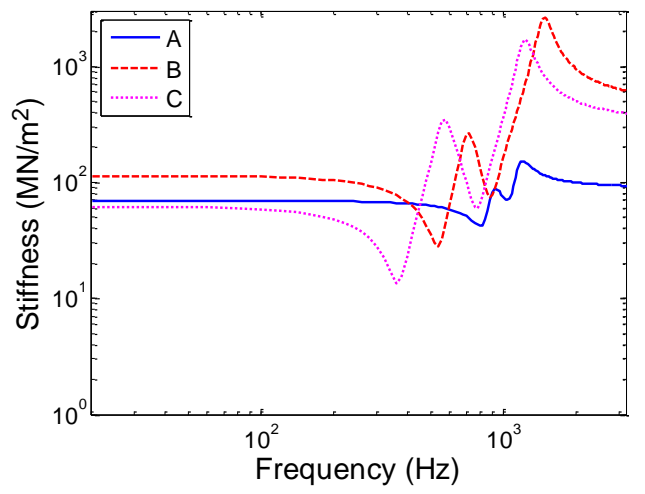

(a)

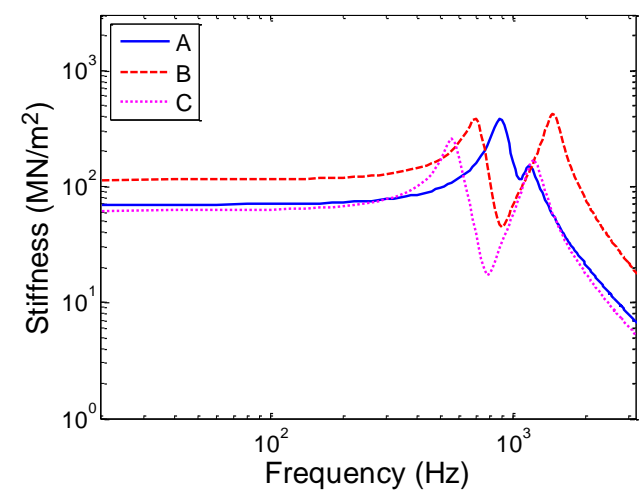

(b)

Figure 7. Absolute value of complex fastener stiffness obtained from 2D model for three track models: (a) driving point stiffness on the top of the rail pad; (b) transfer stiffness (blocked force transmitted per unit displacement on the top of the rail pad)

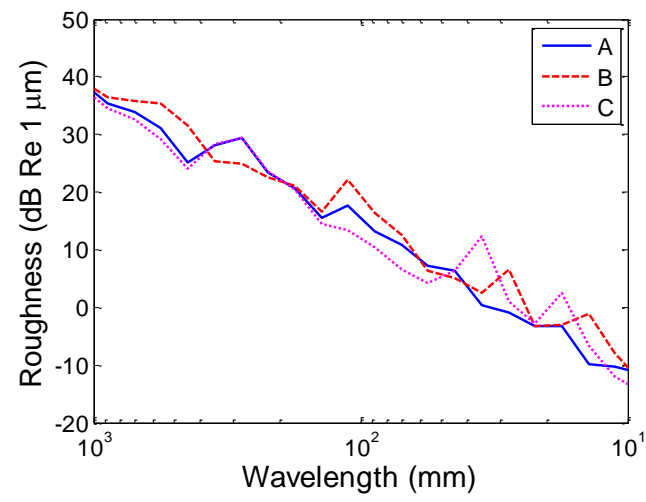

Figure 8. Effective combined roughness estimated with various track models 


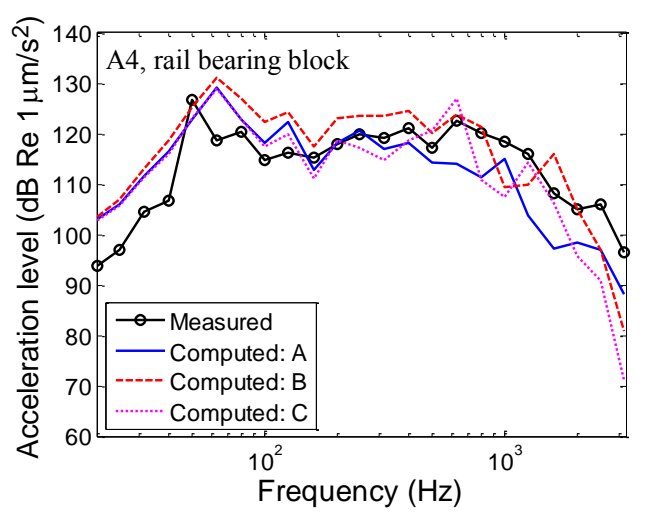

(a)

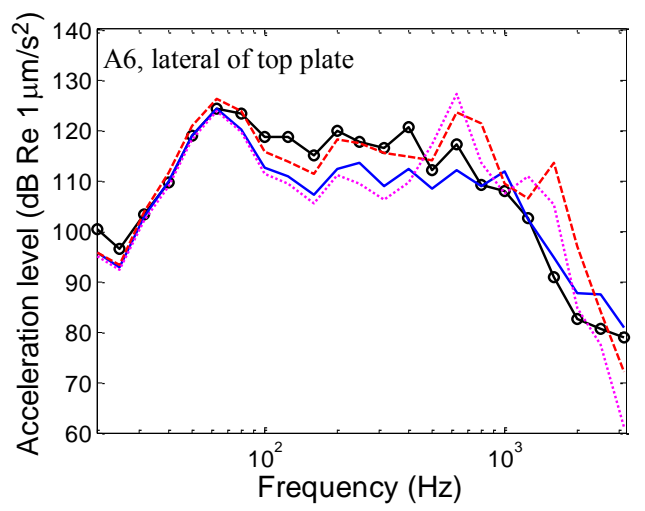

(c)

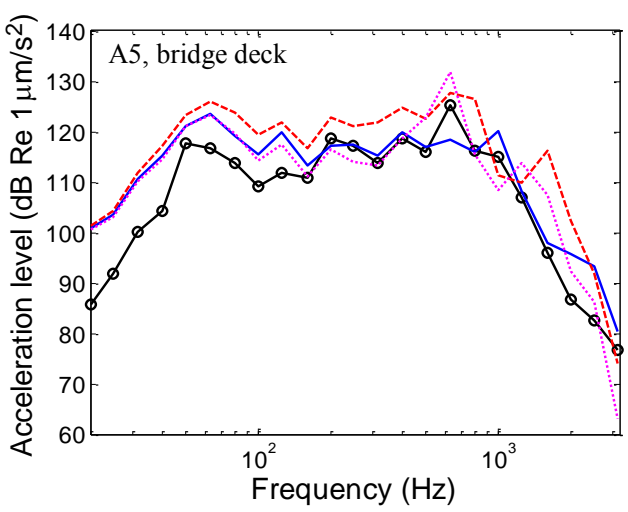

(b)

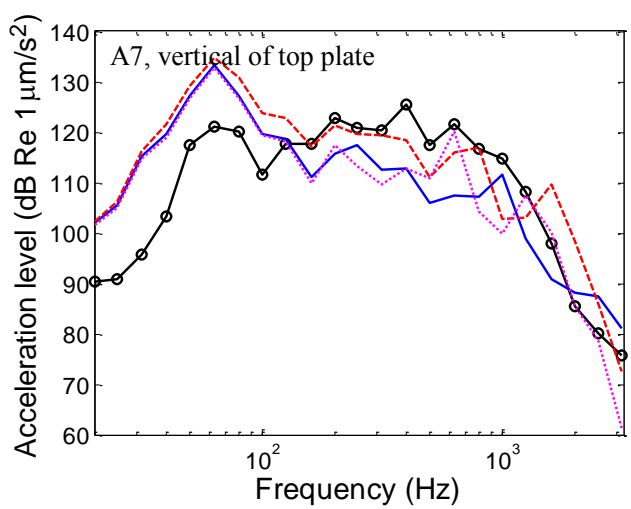

(d)

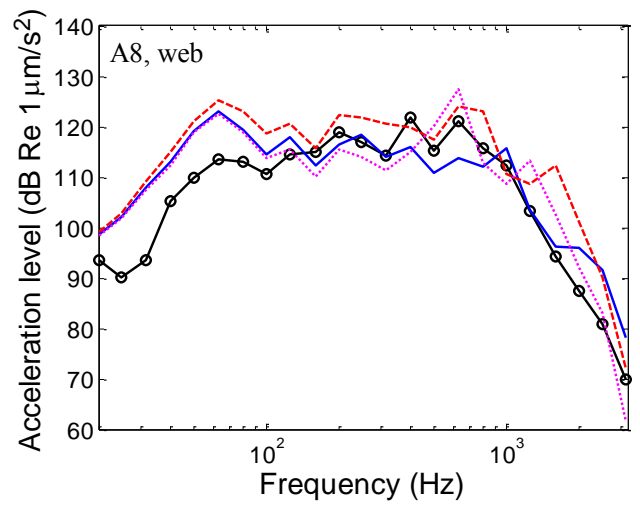

(e)

Figure 9. Measured vibration levels and simulated ones: (a) A4, rail bearing block; (b) A5, bridge deck; (c) A6, lateral of top plate; (d) A7, vertical of top plate; (e) A8, web 


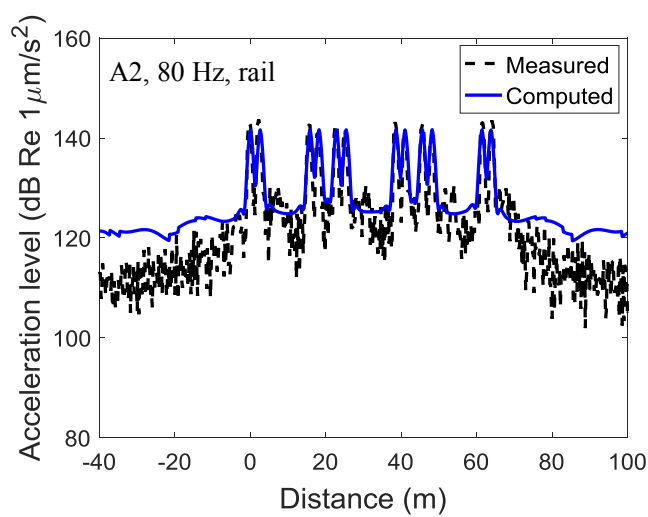

(a)

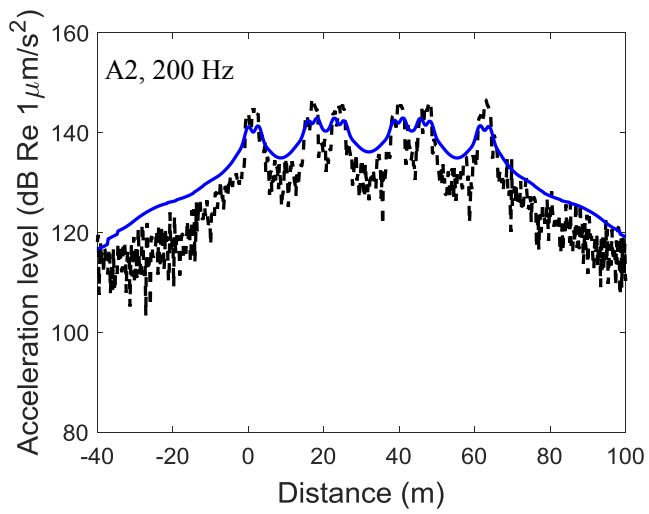

(b)

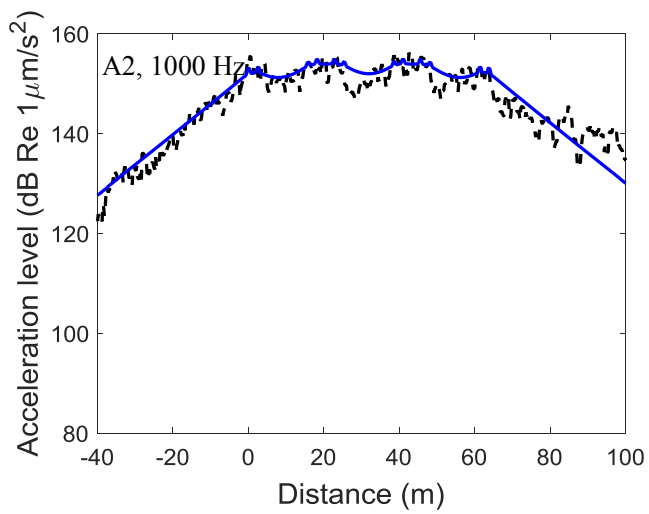

(c)

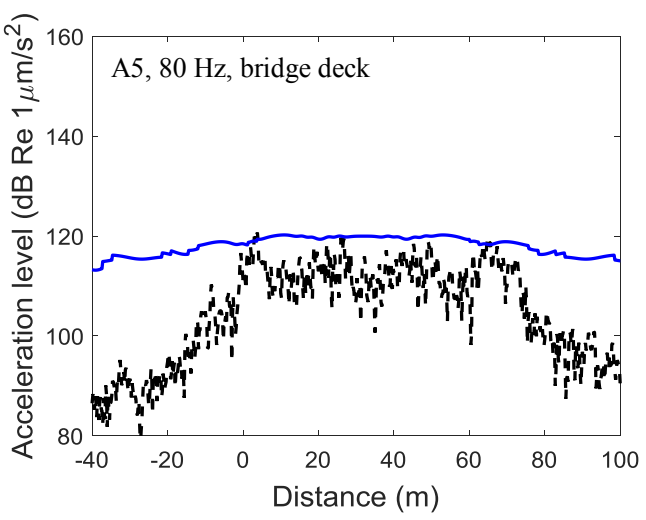

(d)

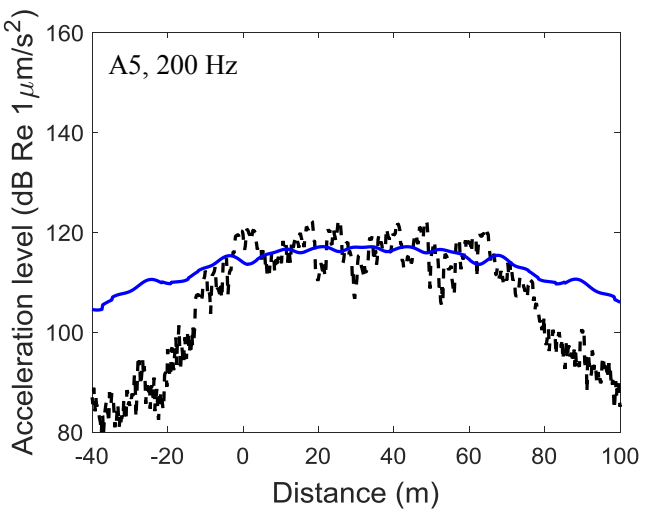

(e)

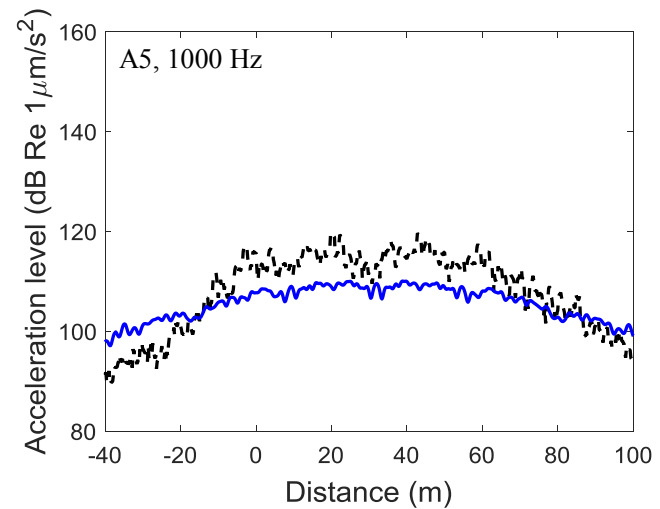

(f)

Figure 10. Instantaneous levels of measured vibration and simulated ones (track set $C$ ) at different one-third octave centre frequencies: (a) A2, $80 \mathrm{~Hz}$; (b) A2, $200 \mathrm{~Hz}$; (c) A2, $1000 \mathrm{~Hz}$; (d) A5, $80 \mathrm{~Hz}$; (e) A5, $200 \mathrm{~Hz}$; (f) A5, $1000 \mathrm{~Hz}$ 


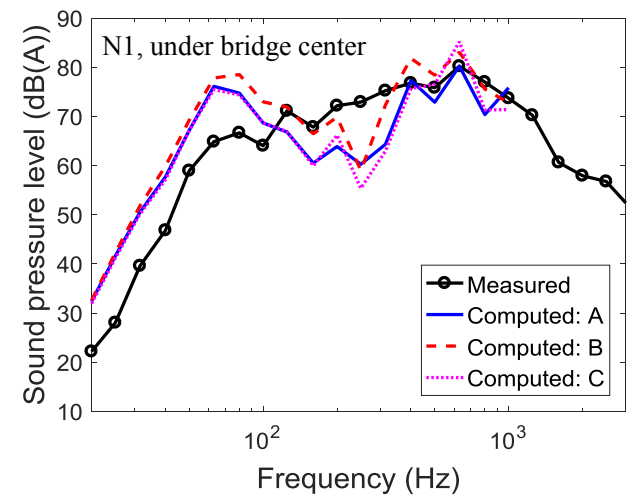

(a)

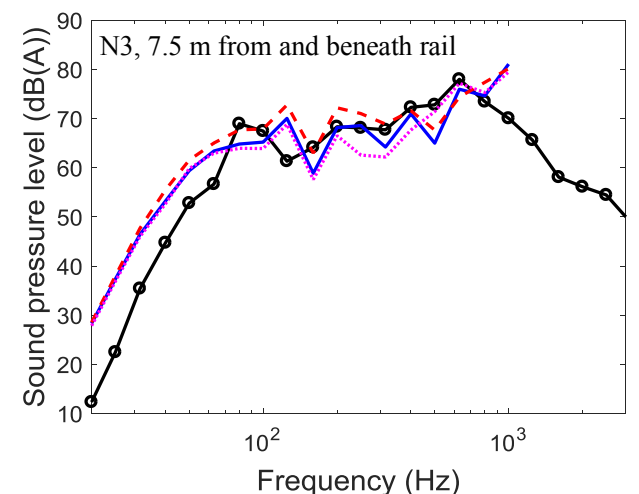

(c)

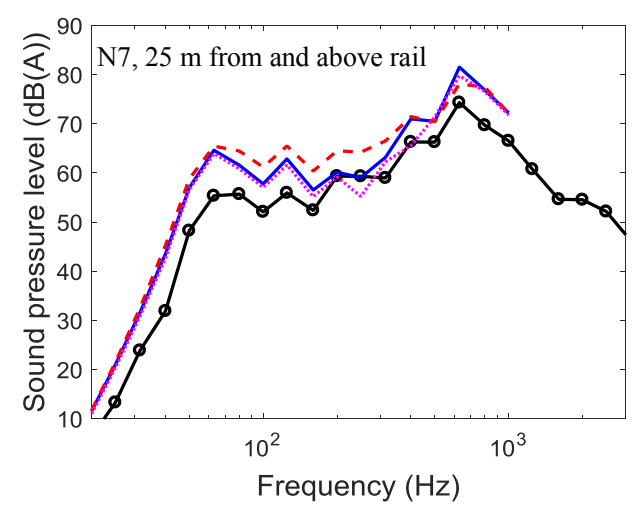

(e)

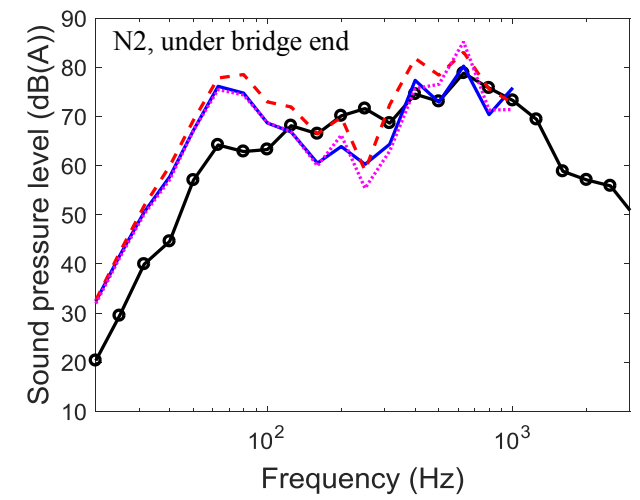

(b)

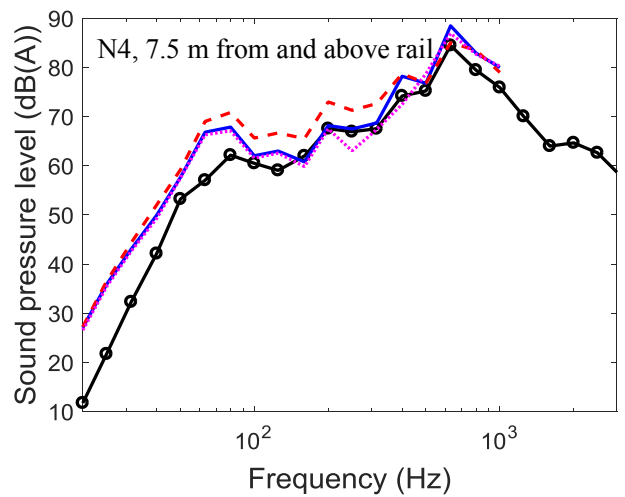

(d)

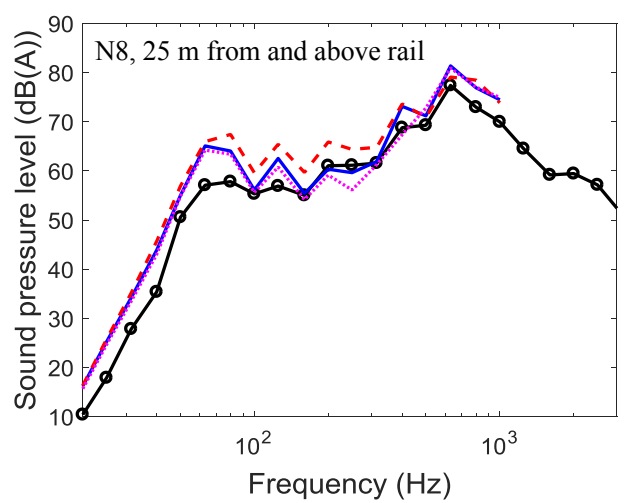

(f)

Figure 11. Measured and predicted total noise levels at various positions: (a) N1, under bridge centre; (b) $\mathrm{N} 2$, under bridge end; (c) N3, $7.5 \mathrm{~m}$ away from and $8.0 \mathrm{~m}$ beneath the rail height; (d) N4, $7.5 \mathrm{~m}$ away from and $1.5 \mathrm{~m}$ above the rail height; (e) N7, $25 \mathrm{~m}$ away from and $1.5 \mathrm{~m}$ above the rail height; (f) N8, $25 \mathrm{~m}$ away from and $4.0 \mathrm{~m}$ above the rail height 


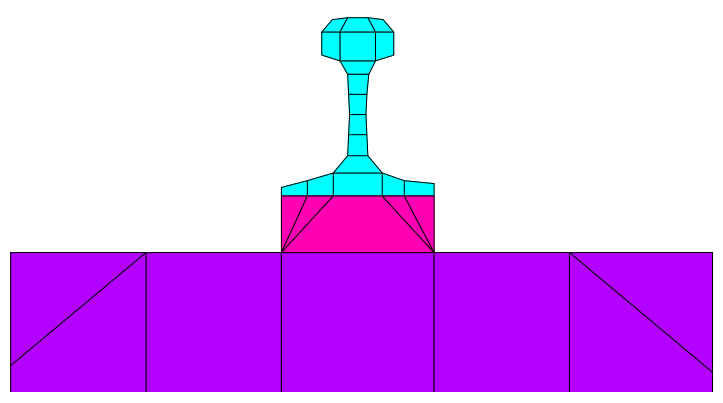

Figure 12. WFE mesh for single-layer fastener model connecting the rail and the bridge

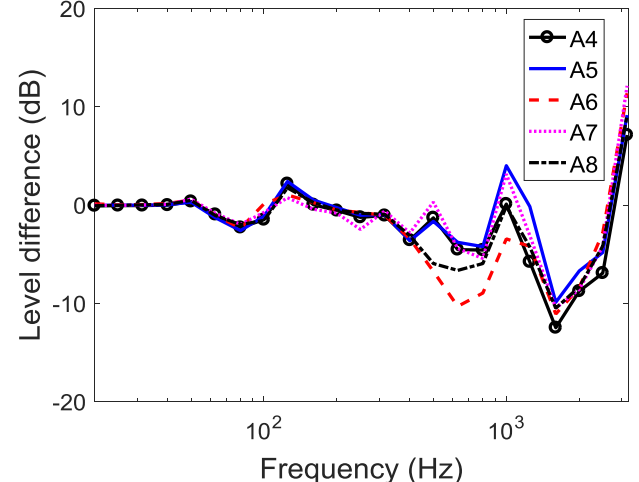

(a)

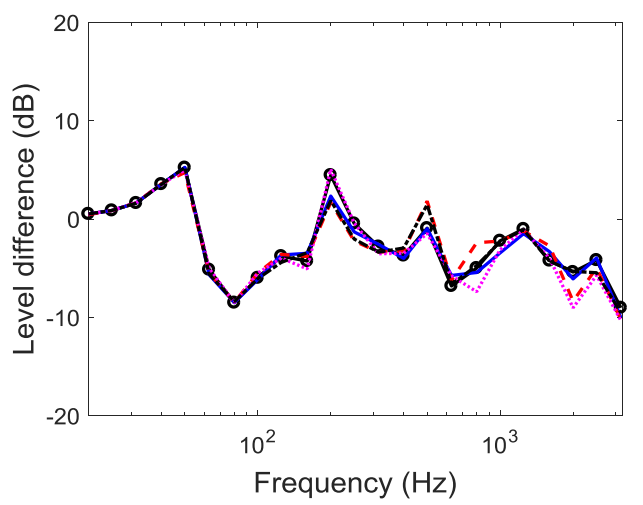

(c)

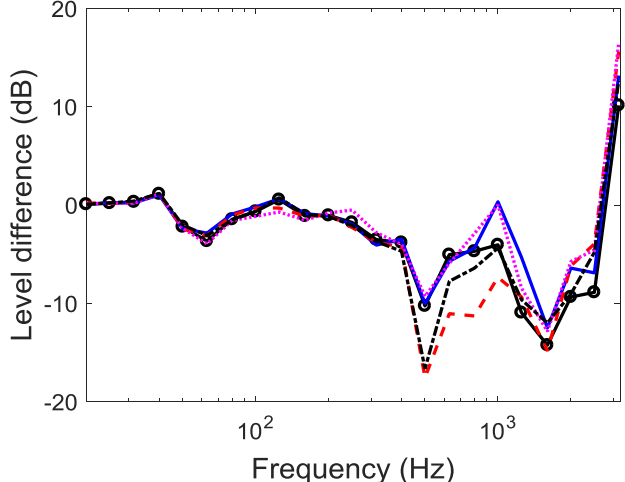

(b)

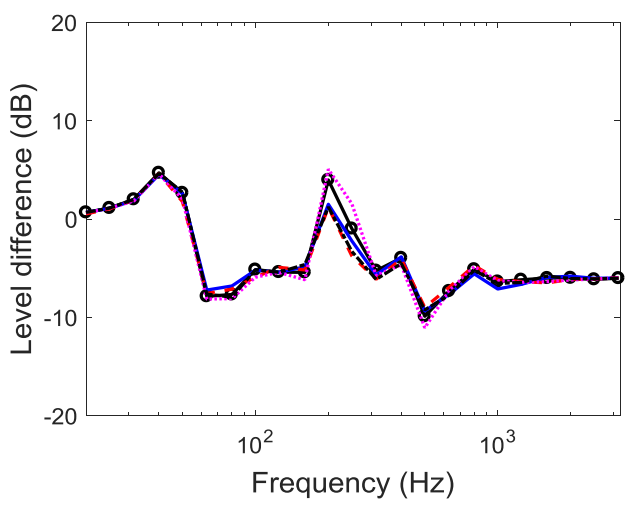

(d)

Figure 13. Difference of bridge vibration levels between various fastener models excited by the same roughness: (a) single-layer fastener model of $132 \mathrm{MN} / \mathrm{m}^{2}$ relative to multi-layer model B; (b) single-layer fastener model of $66 \mathrm{MN} / \mathrm{m}^{2}$ relative to multi-layer model C; (c) multi-layer model C relative to multi-layer model B; (d) single-layer fastener of $66 \mathrm{MN} / \mathrm{m}^{2}$ relative to that of $132 \mathrm{MN} / \mathrm{m}^{2}$ 


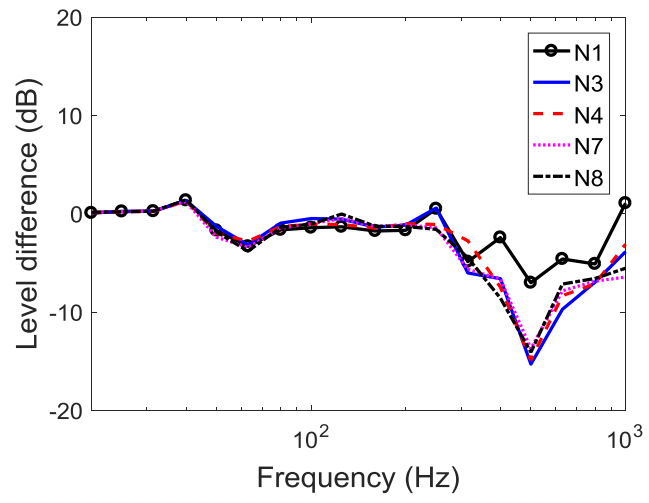

(a)

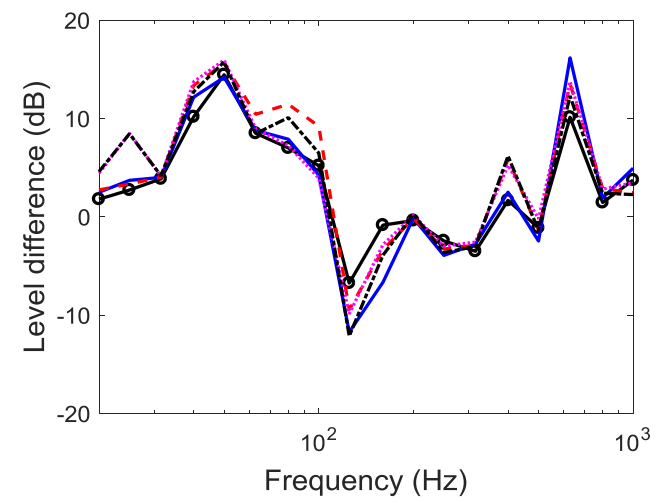

(b)

Figure 14. Difference of bridge noise levels between single-layer fastener model of 66 $\mathrm{MN} / \mathrm{m}^{2}$ and multi-layer model (set $\mathrm{C}$ ) excited by the same roughness: (a) bridge noise; (b) rail noise

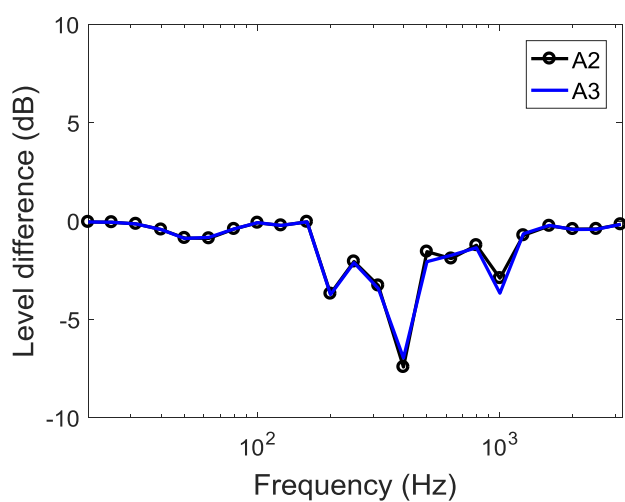

(a)

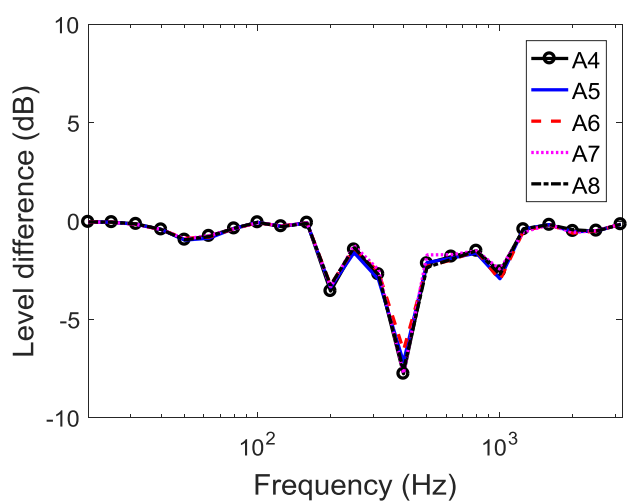

(b)

Figure 15. Changes of vibration levels by neglecting interference of multiple wheels (set C):

(a) rail and baseplate; (b) bridge

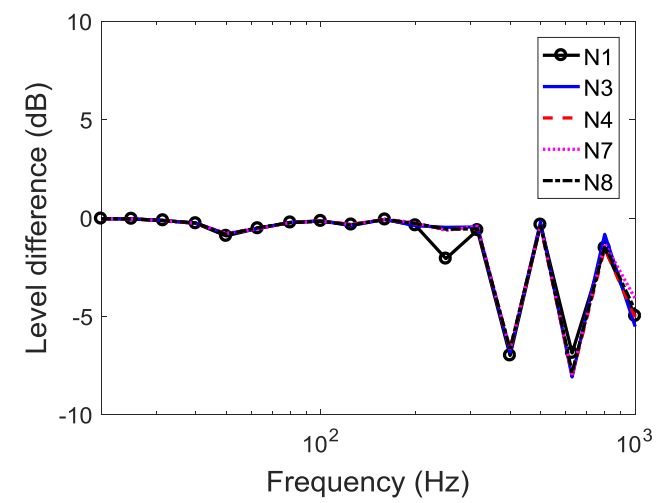

(a)

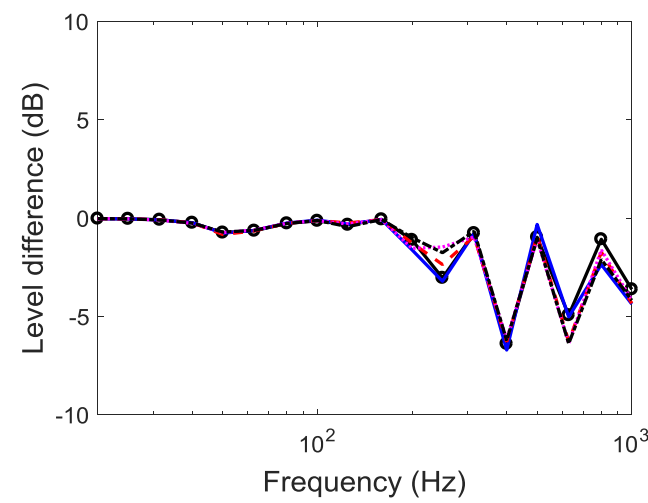

(b)

Figure 16. Changes of noise levels by neglecting interference of multiple wheels (set C): (a) rail noise; (b) bridge noise 


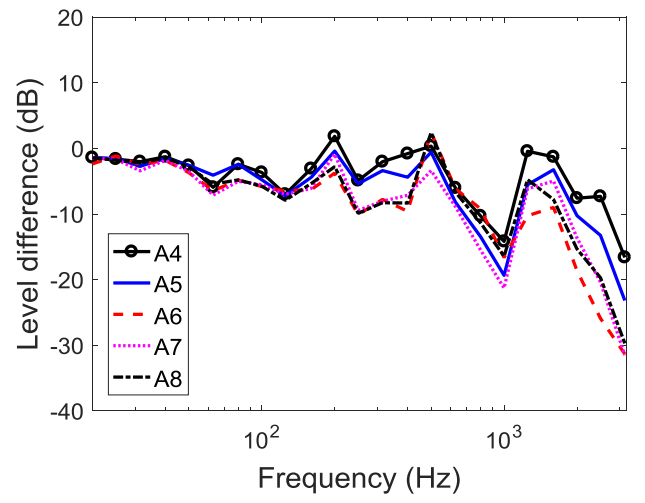

(a)

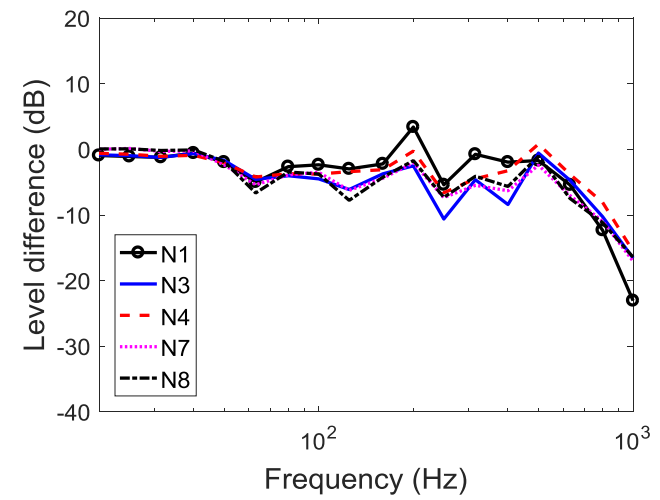

(b)

Figure 17. Changes of bridge vibration and bridge noise levels by increasing the loss factor of the bridge from 0.01 to 0.1 (set C): (a) acceleration; (b) sound pressure

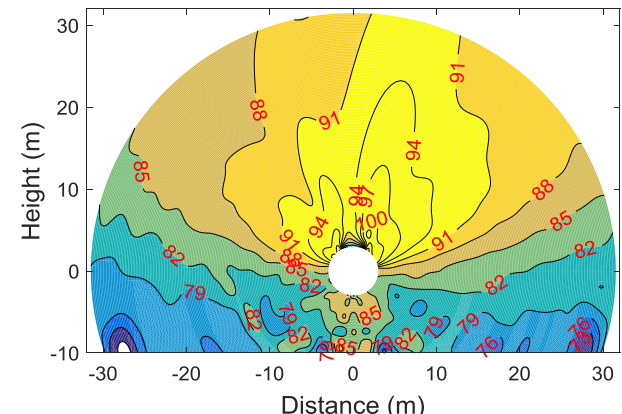

(a)

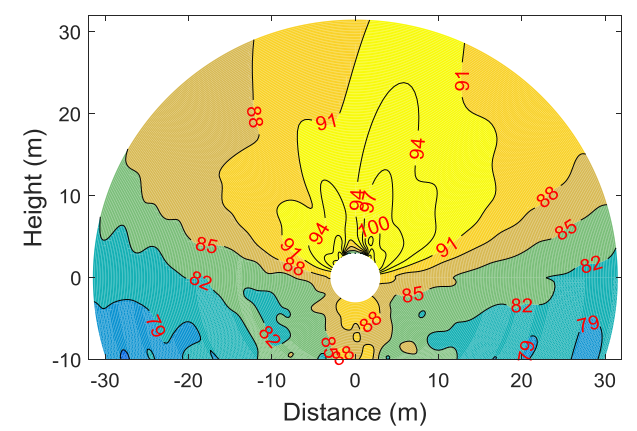

(c)

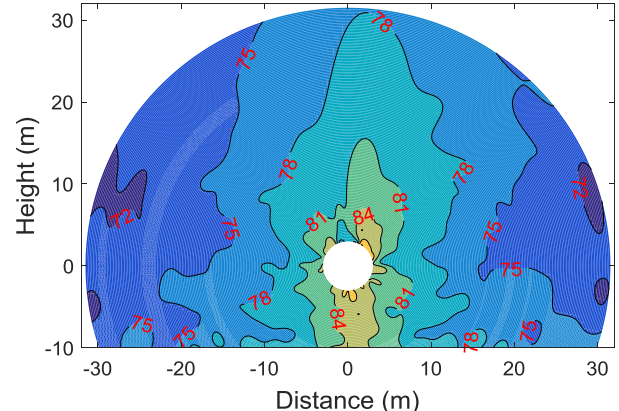

(b)

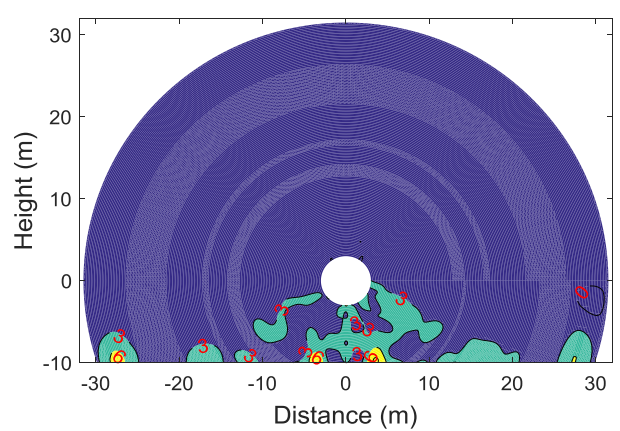

(d)

Figure 18. Contour map of sound pressure levels in $\mathrm{dB}(\mathrm{A})$ at a train speed of $80 \mathrm{~km} / \mathrm{h}$ (set C): (a) rail noise; (b) bridge noise; (c) total noise; (d) total noise subtracting rail noise 